\title{
A Preliminary Formation Flying Orbit Dynamics Analysis for Leonardo-BRDF *
}

\author{
Steven P. Hughes \\ NASA Goddard Space Flight Center \\ Flight Dynamics Analysis Branch, Code 572 \\ Greenbelt, MD 20771 \\ (301) 286-0145 \\ steven.hughes@gsfc.nasa.gov \\ Laurie M. Mailhe \\ a.i. solutions, Inc. \\ Lanham, MD 20706 \\ (301) 306-1756 \\ mailhe@ai-solutions.com
}

\begin{abstract}
Leonardo-BRDF is a new NASA mission concept proposed to allow the investigation of radiative transfer and its effect on the Earth's climate and atmospheric phenomenon. Enabled by the recent developments in small-satellite and formation flying technology, the mission is envisioned to be composed of an array of spacecraft in carefully designed orbits. The different perspectives provided by a distributed array of spacecraft offer a unique advantage to study the Earth's albedo. This paper presents the flight dynamics analysis performed in the context of the Leonardo-BRDF science requirements. First, the albedo integral is investigated and the effect of viewing geometry on science return is studied. The method used in this paper, based on Gauss quadrature, provides the optimal formation geometry to ensure that the value of the integral is accurately approximated. An orbit design approach is presented to achieve specific relative orbit geometries while simultaneously satisfying orbit dynamics constraints to reduce formation-keeping fuel expenditure. The relative geometry afforded by the design is discussed in terms of mission requirements. An optimal Lambert initialization scheme is presented with the required $\Delta V$ to distribute all spacecraft from a common parking orbit into their appropriate orbits in the formation. Finally, formation-keeping strategies are developed and the associated $\Delta V$ 's are calculated to maintain the formation in the presence of perturbations.
\end{abstract}

*U.S. Government work not protected by U.S. copyright.
TABLE OF CONTENTS

\author{
1. INTRODUCTION \\ 2. SCIENCE REQUIREMENTS \\ 3. FORMATION DYNAMICS \\ 4. Formation PERFormanCE \\ 5. $\triangle \mathrm{V}$ BUDGET \\ 6. ALTERNATIVE FORMATION \\ 7. CONCLUSIONS
}

\section{INTRODUCTION}

Recently, the concept of spacecraft formation flying has received significant attention from the space systems community. Although it is not a new concept, new realizations have been made in the benefits of a distributed array for many science missions. The multiple perspectives provided by an array of spacecraft provide a platform to perform missions not possible with single-spacecraft missions. Yet, there are many scientific and engineering challenges which must be overcome before such missions become operational.

Scientists at NASA's Goddard Space Flight Center(GSFC) are currently considering the possibility of using a formation of spacecraft to study atmospheric phenomenon. The instantaneous net radiation flux, or the albedo, at the top of the atmosphere is one of the primary drivers of climate and global change [1]. A distributed array of spacecraft provides a unique approach to estimate the net flux. However, the array geometry must be consistent with the laws of dynamics and satisfy requirements imposed by the science mission. 
This paper presents the preliminary flight dynamics analysis performed in context of the science requirements to effectively estimate the albedo effect. We begin with an introduction to the science of the Bi-Directional Reflectance Distribution Function(BRDF). The reference frames chosen for the analysis are described and the equation for the radiative forcing is presented. Subsequently, we present a method for estimating the albedo using a finite number of measurements. The technique is based on Gauss quadrature. From the study of the albedo integral, we determine the orbit dynamics requirements to accomplish the science objectives. These include the relative geometry of the spacecraft in formation as well as the reference orbit characteristics.

A significant portion of the paper is devoted to orbit design. Reference [2] presented a strategy to design relative orbits that are " $J_{2}$ invariant" by requiring that the node rates and argument of latitude rates for the reference and companion orbits be equal. Reference [3] developed an algorithm to design ring formations and determined a correction technique to lessen the degeneration of the geometry due to along-track secular effects of $J_{2}$. Yet, little work is found in the literature on methods that mitigate perturbation effects through careful orbit design and achieve a specific formation geometry. Although the formation geometry cannot be maintained in the presence of disturbing perturbations without an active control system, it is possible to design the orbits to reduce the amount of control effort required. The primary perturbation causing formation decomposition is $J_{2}$. The effects of $J_{2}$ can be broken down into specific effects on the orbital motion. In this work, we identify which affects cause a change in the formation geometry and which affects, assuming a careful orbit design, will not degrade the relative motion. We present a strategy to diminish the effects of $J_{2}$ on orbit evolution while simultaneously satisfying constraints on the formation geometry imposed by the science requirements.

In order to ascertain the feasibility of the approach, we present a preliminary $\Delta \mathrm{V}$ budget analysis. We assume that all spacecraft will be launched on a single vehicle. Therefore, the spacecraft must be relocated from a common parking orbit into their appropriate final orbit. This is further complicated by timing constraints to insure that the desired relative positions are achieved. The $\Delta V$ 's are obtained by finding the optimal twoimpulsive-burn transfer orbit that meets the timing constraints to achieve the desired relative positions. A similar strategy, based on work by Chao et.al.[4], is used to determine the $\Delta \mathrm{V}$ budget for formation-keeping in the presence of perturbations.

\section{SCIENCE REQUIREMENTS}

In this section we introduce the science of BRDF and the definition of radiative forcing used in this analysis. First, we present a conceptual description of BRDF. The coordinate system and the variables used to describe radiative forcing are defined. A mathematical model is presented along with a Gauss quadrature technique for obtaining accurate approximate solutions. Based on the solutions, we present orbit requirements to meet the science objectives.

\section{What is BRDF?}

The Bi-Directional Reflectance Distribution Function (BRDF) characterizes the variation of reflected sunlight from a given surface. The BRDF varies with surface (smoothness etc.) and optical properties (transmission, reflection, absorption, emission etc.), and the wavelength of radiated light. The BRDF also depends on the angle at which the light impacts the surface (illumination geometry) and on the angle at which you look at the surface (viewing geometry). An intuitive example of BRDF is provided by considering a recently mowed field of grass. The blades of grass tend to lie in the direction of the motion of the mower. The affect of the mowing direction is easily observed with the human eye. The stripes in the field appear because the grass is leaning in the direction in which it was mowed. Different amounts of light are reflected depending upon the direction of the blades of grass. However, the complexity of BRDF is not completely portrayed in this simple example. The effects of BRDF extend into the non-visible spectrum and depend on the position of the sun, atmospheric conditions, and the type of surface. On large scales, BRDF has a significant effect on climate phenomenon because it determines how much energy is radiated back into space and how much is retained in the atmosphere. In this study, we are interested in the total net radiation flux from a given region. In other words, we are interested in the integral of BRDF over the entire viewing geometry space for a particular subject. We will call this integral the "radiative forcing", although in the science community, the term is used slightly differently.

For Leonardo-BRDF, we are interested in the radiative forcing of targets of interest such as, clouds, ocean surfaces, and various types of land surfaces. Although considerable efforts have been spent on radiative flux measurements, the errors are too large for many climate applications. According to Wielicki et al. [5], the biggest instantaneous flux errors in past and current satellite measurements come from poor knowledge of the angular variation of Earth-leaving radiance. These angular errors range from $12.5 \mathrm{~W} / \mathrm{m}^{2}$ for the ideal Clouds and the Earth's Radiant Energy System (CERES) to $37.5 \mathrm{~W} / \mathrm{m}^{2}$ for the Earth Radiation Budget Experiment (ERBE), and account for over $90 \%$ of the total error from all sources. These errors are an order of magnitude larger than typical climate forcings (e.g. 4 $\mathrm{W} / \mathrm{m}^{2}$ for doubled $\mathrm{CO} 2$ ). In order to improve climate modeling with radiative forcing effects the albedo must be measured to within $0.5 \%$ accuracy.

Before introducing the physics of BRDF and radiative forcing, we define the reference frame for the BRDF analysis. The origin of the BRDF frame, $\mathcal{F}_{B}$, is a target located at the top of the atmosphere (TOA). An illustration of $\mathcal{F}_{B}$ is shown in Fig. 1. We assume TOA to be at an altitude of $30-\mathrm{km}$. The location of 
the target is defined as the nadir point of the reference spacecraft and will be discussed in more depth in a later section. We define the $z$-axis as the normal to the Earth's surface in the radial direction of the reference spacecraft. The principal plane is defined as the plane formed by the Sun, the point being viewed and the reference spacecraft. For convenience, we choose the $x$-axis to lie in the principal plane and the $y$-axis completes the right hand set.

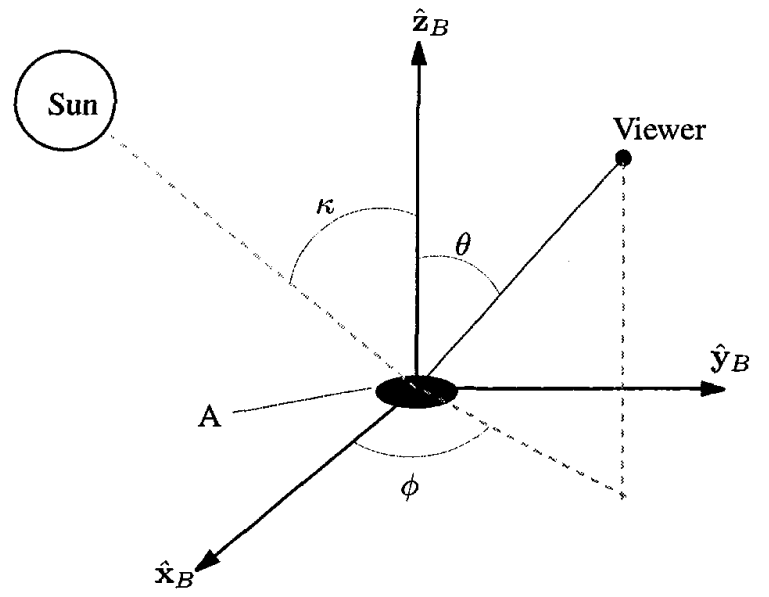

Figure 1: Viewing Geometry and Frame $\mathcal{F}_{B}$

Returning to Fig. 1, we define the view zenith angle, $\theta$, as the angle between the line of sight of the viewer and the $\hat{\mathbf{z}}_{B}$-axis. Similarly, the sun zenith angle, $\kappa$ is defined as the angle between the sun vector and the $z_{B}$-axis. The view azimuth angle, $\phi$, is defined as the angle between the projection of the line of sight in the $x y$-plane and the $x$-axis. The BRDF frame rotates according to the position of the Sun with respect to the target. Therefore, the azimuth angle evolution in the BRDF frame is highly non-intuitive, which will affect our future approach to the orbit design. The orbit design has been performed in an intermediate reference frame discussed later. We show in a later section that only the relative spacing of the spacecraft in $\phi$ is important. Thus, we can examine, without loss of generality, the evolution of the azimuth angle in a frame that does not rotate about the $z_{B}$-axis.

With the definitions of the coordinate systems and variables in hand, we can now investigate the mathematical model for the radiative forcing.

\section{How Do We Estimate the Radiative Forcing?}

The radiative forcing is the integral of BRDF over the entire viewing geometry space of $\phi$ and $\theta$. Furthermore, the albedo is also dependent on the area of the pixel, $A$, and the sun zenith angle, $\kappa$. To simplify the expression for the flux integral we define a new variable $\mu$, given by

$$
\mu=\cos \theta
$$

The exact expression for the instantaneous flux for a pixel of area $A$ is expressed as

$$
F=\int_{A} \int_{0}^{2 \pi} \int_{0}^{1} \mu I_{A}(\mu, \phi, \kappa, \lambda) d \mu d \phi d A
$$

where $I_{A}$ is the radiance from the TOA pixel in the direction of $\theta$ and $\phi$. In this expression $\kappa$ and $\lambda$ have been included in the flux expression as a reminder that the flux is dependent on the illuminating geometry and the wavelength of light being investigated. In subsequent expressions, $\kappa$ and $\lambda$ are left out of the expression without loss of generality. However, it must be remembered that although there is no integration over $\kappa$ and $\lambda$ a particular flux value is dependent on them. The flux value is assumed to be for a particular wavelength of light and a specific illuminating geometry.

To obtain the exact measure of the flux, measurements must be available from all possible viewing perspectives. In practice, such information is not available. Therefore, methods must be developed to accurately estimate the radiance integral in Eq. (2) using a finite number of measurements. Generally, the more spacecraft in the array, the more accurate the approximation will be. Yet, we need to minimize the number of spacecraft for practical reasons. In order to estimate the flux integral, we separate the $\theta$ and $\phi$ dependency using common radiative transfer theory and expand the radiance $I_{A}$ in Fourier series:

$$
\begin{array}{r}
I_{A}(\mu, \phi)=\sum_{m=0}^{M}\left[I_{m}^{(1)}(\mu) \cos m \phi+\right. \\
\left.I_{m}^{(2)}(\mu) \sin m \phi\right]
\end{array}
$$

For 1-Dimensional radiative transfer, the sine terms vanish. Furthermore, when the right-hand side is integrated over $\phi$ the only remaining term is $I_{0}^{(1)}$. Hence, the integral over $\mu$ takes the form

$$
\int_{0}^{1} I_{0}^{(1)}(\mu) d \mu=\sum_{i=1}^{N} w_{i} I_{0}^{(1)}\left(\mu_{i}\right)
$$

where we approximate the integral with a quadrature sum with weights $w_{i}$ and abscissae $\mu_{i}$. There are several techniques for determining the optimal values of $w_{i}$ and $\mu_{i}$ including Gauss quadrature and Chebychev equal weight quadrature [6]. If the spacecraft are located at the ideal abscissae, then the spacecraft form a quadrature array. Each measurement is then summed up to obtain the flux at a given instant where $\mathrm{N}$ is the total number of spacecraft.

The current formation design attempts to demonstrate the feasibility of selecting spacecraft orbits that follow a set of quadrature rules. The abscissae values depend on the choice of quadrature rule chosen. In this work, we have investigated several types but continue the analysis without reference to a specific set of abscissae. Yet, it is important to discuss some trends seen in the solutions using the Gauss and Chebychev equal 
weight quadrature approaches. Most importantly, both techniques require abscissae values, $\mu_{i}$, near 0 and near 1 . From Eq. (1) we see that this results in zenith angles near, but not equal to zero, and near, but not equal to $\pi / 2$. Therefore, we must design the formation to provide these extremes in viewing geometry. Generally, a formation will not be able to provide viewing geometries that exactly satisfy the quadrature analysis. The following analysis attempts to ascertain to what degree we can achieve a quadrature array formation.

With a basic understanding of BRDF, we are now prepared to discuss the specific orbit requirements in light of the science objectives and the formation geometry required to estimate the flux integral.

\section{What are the Orbit Requirements?}

The orbital requirements originate from three main areas: the quadrature analysis, BRDF symmetry properties and general formation flying dynamics constraints. The Gauss quadrature analysis requires specific viewing geometries, which place constraints on the relative positions of the spacecraft in formation. Symmetry properties of the BRDF integral can be used to our advantage by imposing further constraints on the reference orbit. Finally, there are general formation flying concerns that are addressed in Section 3.

Perhaps the most challenging constraints in the context of Leonardo are those resulting from the quadrature analysis. To accurately estimate the radiative transfer, measurements must be taken at specific positions with respect to the BRDF reference frame. This places stringent requirements on the relative positions of spacecraft in formation. First, to obtain an accurate average of the azimuthal dependence, the spacecraft must be spaced nearly equal in azimuth. The zenith angle requirement is even more stringent. Depending on the type of quadrature solution, the spacecraft must be permanently located at specific values of the view zenith angle.

By taking advantage of symmetry principles, we can obtain "extra" data points to increase the accuracy of the approximation. If the surface being investigated is assumed homogeneous, the BRDF is symmetric about the principal plane. Therefore, data points on one side of the principal plane can be reflected to the other side. By using a Sun-synchronous reference orbit with a noon-equator crossing, the reference orbit is in the principal plane, which can greatly reduce the amount of post-processing required to perform

There are further considerations for using a Sun-synchronous reference orbit. By definition, the orbit plane rotates about the Earth at the same rate as the Earth rotates about the Sun. Therefore, the reference plane maintains a constant geometry with respect to the Sun. The result is that only half of the orbit can view the sun-lit side of the Earth. This condition will be maintained for the entire mission lifetime. Therefore, we are free to design the orbits such that only the portion that experiences daylight provides a useful geometry. This will prove to yield some interesting results. On the other hand, for Sunsynchronous orbits there is a coupling between the sun position and latitude. A given latitude will always experience the same illuminating geometry. This limits the amount of understanding that can be gained on the albedo effect for different illuminating geometries. However, if the orbit is not chosen to be Sun-synchronous then the orbit planes will rotate with respect to the sun. Eventually the portion of the orbit that was on the dark side of the Earth will rotate into the Sun-lit side. Therefore, the entire orbit must be designed to provide a useful geometry. This will prove to be very difficult.

The nominal mission for Leonardo-BRDF assumes that the "target" or point of interest lies at the nadir of the reference spacecraft. Yet, it is likely that there will often be targets of interest that are within the formation field of view that do not lie on the reference nadir. Eventually, we need to develop an approach to determine the ability of the formation to take useful measurements of off-nadir targets. Yet, time constraints have not permitted a rigorous pursuit of this approach and it is a topic of future research.

In summary, the orbit requirements originate from three main areas: the quadrature analysis, the BRDF symmetry properties and general formation flying constraints. The quadrature approach requires specific viewing geometries, which place constraints on the relative positions of the spacecraft in formation. Further complications arise when off-nadir targets are of interest. Symmetry properties of the BRDF integral can be used to our advantage by imposing further constraints on the reference orbit. Choosing a Sun-synchronous orbit has numerous advantages. Finally, there are general formation flying concerns that are addressed in Section 3. The orbit requirements imposed by the science objectives are:
- Maintain specific view zenith angles
- Provide near equal spacing in azimuth
- Use a Sun-synchronous reference orbit
- Provide a noon equator-crossing
- Consider only the Sun-lit portion of the Earth

In the next section, we discuss some general formation flying dynamics concerns and develop the orbits that provide the necessary relative dynamics to perform BRDF measurements.

\section{FORMATION DYNAMICS}

We begin this section by presenting some convenient coordinate systems and some challenges of orbit design to minimize the adverse effects of perturbations. Next, we develop the formation dynamics. The approach allows the analyst to specify a specific formation geometry and the resulting formation satisfies dynamical constraints to reduce $J_{2}$ perturbation effects. Finally, we discuss the physics of the allowable solutions 
and compare the non-linear method with a Hill's equations approach.

Three coordinate systems are used in the development of the formation dynamics. An illustration of the frames is shown in Fig. 2. The first frame is similar to the ones used by Hill [7] and later by Clohessy and Wiltshire [8] in their original studies of relative motion. We denote the coordinate system $\mathcal{F}_{H}$. The origin of $\mathcal{F}_{H}$ is located at the reference spacecraft. The $x_{H^{-}}$axis is in the radial direction, the $z_{H}$-axis is in the orbit normal direction, and the $y_{H}$-axis completes the right handed set. Note that the frame is not inertial because it rotates depending on the orbit of the reference spacecraft. The term "in-plane" refers to the projection of the relative motion in the $x y$-plane. Similarly, the terms "along-track" and "cross-track" are used to describe motion in the $y_{H}$ and $z_{H}$ directions respectively.

The second frame used in the analysis is the common Earthcentered inertial frame, $\mathcal{F}_{I}$. The origin of $\mathcal{F}_{I}$ is the center of the Earth. The $x_{I}$-axis points towards the vernal equinox and the $z_{I}$-axis extends through the North Pole. The $y_{I}$-axis is chosen to complete the right-handed set.

The third and final coordinate system is denoted $\mathcal{F}_{R}$. The frame is fixed in inertial space with its origin at the center of the Earth. However, the $x_{R}-y_{R}$ plane is not the Earth's equatorial plane, it is the orbital plane of the reference circular orbit. The $x_{R}$-axis is defined by the line of relative nodes of the reference and a particular companion orbit. The $z_{R}$-axis is normal to the reference plane of motion and the $y_{R}$-axis completes the right-handed set. Defining $\mathcal{F}_{R}$ in this manner allows some useful algebraic simplification that is demonstrated in a later section. Properties associated with the reference orbit are given with the subscript " $r$ ". The remaining orbits composing the formation are termed "companion" orbits and there associated properties are shown with the subscript " $c$ ".

Many formidable complications are caused by orbit perturbations. The dominant perturbation, and the only one considered in this study, is $J_{2}$. In general, $J_{2}$ causes periodic changes in all of the orbital elements. However, only the right ascension of the ascending node, $\Omega$, the argument of periapsis, $\omega$, and the mean anomaly, $M$, experience secular drifts. To first order in $J_{2}$, the secular drift rates are given by

$$
\begin{gathered}
\dot{\Omega}=-\frac{3 n R_{e}^{2} J_{2}}{2 p^{2}} \cos i \\
\dot{\omega}=-\frac{3 n R_{e}^{2} J_{2}}{4 p^{2}}\left(4-5 \sin ^{2} i\right) \\
\dot{M}=n+\frac{3 n R_{e}^{2} J_{2} \sqrt{1-e^{2}}}{4 p^{2}}\left(3 \cos ^{2} i-1\right)
\end{gathered}
$$

Foremost, we must design the formation to provide the necessary relative geometry to accomplish the science. However, the $J_{2}$ perturbation will cause the formation geometry to degenerate rapidly. Although formation geometry cannot be maintained in the presence of disturbing perturbations without an active control system, it is possible to design the orbits to reduce the amount of control effort required. In the next few subsections, we explore some techniques that simultaneously provide a desired formation geometry and mitigate the effects of the secular $J_{2}$ perturbations. For reasons to be explained later, we enforce the constraints

$$
\begin{aligned}
\dot{\Omega}_{r} & =\dot{\Omega}_{c} \\
\dot{M}_{r} & =\dot{M}_{c}
\end{aligned}
$$

The results are applicable to problems beyond the scope of Leonardo. Therefore, the solutions are presented in a general setting. Their implications for Leonardo are presented in a separate section.

\section{Rotating Formation Design}

The formation is developed by considering the projection of the actual motion onto the $y_{H}-z_{H}$ plane. A circular reference orbit is assumed. We investigate both linear and non-linear techniques to develop the orbits. For the non-linear approach, we characterize the size of the formation by using the angular, as opposed to the spatial, separations of the spacecraft where the vertex of the angular separation is the center of the Earth. A technique is developed to produce an ellipse-like projection of the relative motion in the $y_{H^{-}} z_{H}$ plane. An illustration of several different types of projections is shown in Fig. 3. We wish to obtain relative motion projections of Type 1 . This can be achieved by applying the simple dynamical constraints:

$$
\dot{y}_{H}=0 \quad \text { when } \quad z_{H}=0
$$

We refer to these constraints as the turn-around conditions.

We begin the investigation with some preliminary relative motion considerations. The dimensions of the relative motion are determined in terms of the eccentricity and relative inclination of the planes of motion. Next, we consider the implication of the turn around conditions seen in Eq. (10) on the orbit dynamics and the constraints to reduce $J_{2}$ effects. Some second order analytic results are presented; however, the equations can be solved numerically to higher order using the second order approximation as an initial guess. The section is concluded with a discussion of the four solutions that satisfy all of the constraints and a linear approach based on Hill's equations [7] is developed and compared to the non-linear approach.

The angles $\alpha_{y}$ and $\alpha_{z}$ are defined as the maximum angular separations in the $y_{H}$ and $z_{H}$ directions, respectively. They are the angular equivalents to the amplitudes in Hill's equations. The vertex of the angles is the center of the Earth. The out of plane angular amplitude, $\alpha_{z}$, is entirely dependent on the relative inclination, $\Delta i$, of the two planes of motion. The 


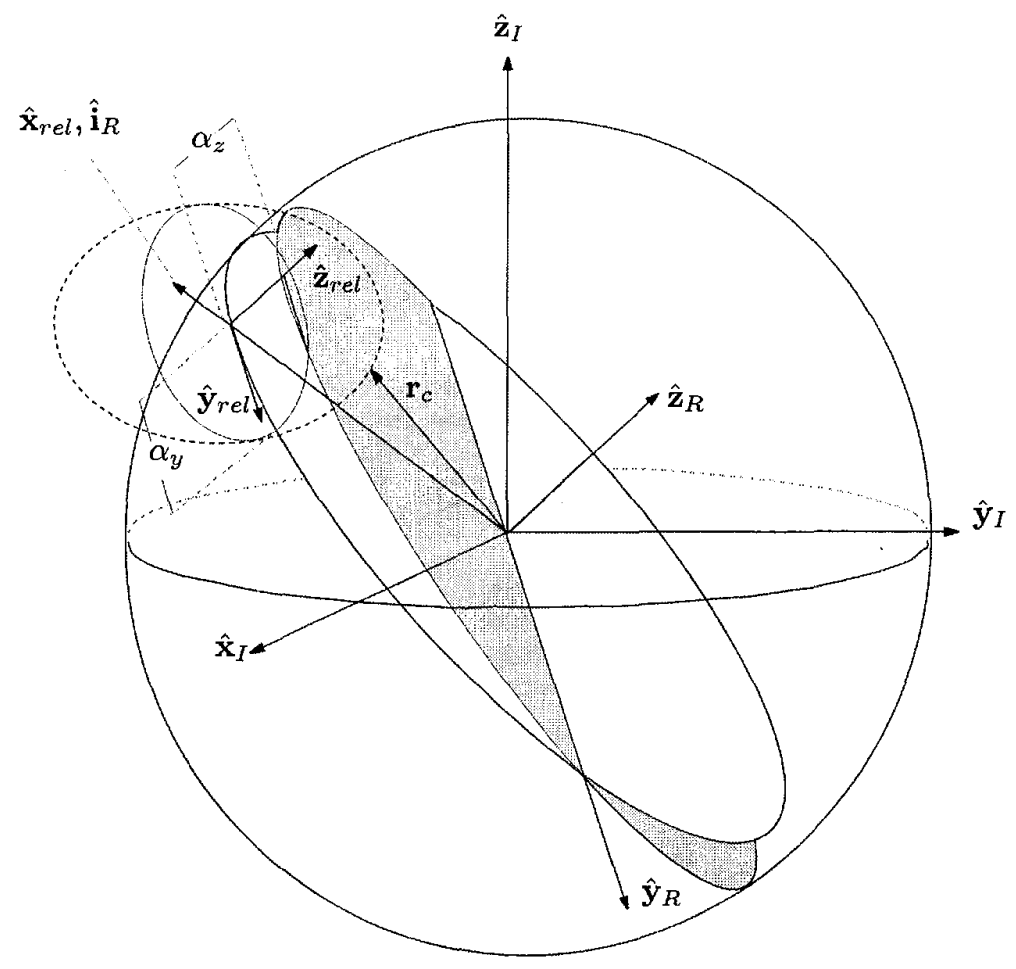

Figure 2: Reference Frames and Formation Geometry

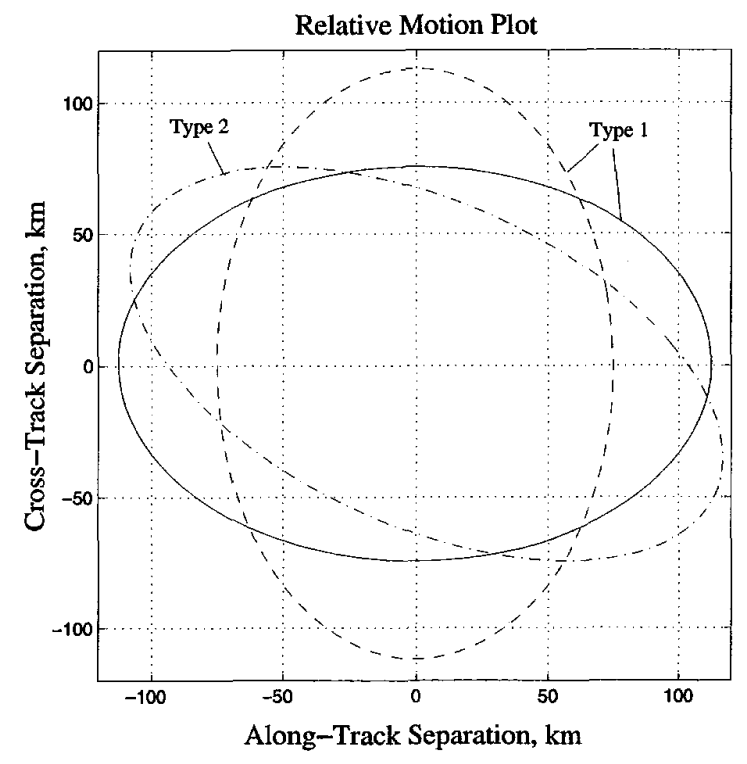

Figure 3: Relative Motion Description relative inclination is defined to be the angle between the orbit normal's of the reference and a particular companion orbit. To satisfy the orbit constraints presented later, we must position the companion orbit at a specific inclination. For a given reference orbit, it is possible to choose the companion inclination and RAAN to obtain the desired across-track amplitude while satisfying the constraints. However, this approach does not work for equatorial orbits.

We wish to determine the dimensions of the relative orbit in terms of the orbital elements of the companion orbit. The plane of motion of the reference orbit is assumed known and the goal is to calculate the elements of the companion orbit to meet the geometry and orbit constraints. The argument of latitude, $u_{r}$, of the reference spacecraft in the reference plane of motion is not yet specified. By choosing $u_{r}$ carefully the formation design can be greatly simplified.

A view of the relative motion geometry is shown in Fig. 4. By definition, the reference spacecraft is always at the origin of $\mathcal{F}_{H}$ and is defined by the vector $\mathbf{r}_{r}$. The vector $\mathbf{r}_{c}$ describes the position of an arbitrary companion spacecraft. The quantity $\psi$ is the along-track component of angular separation of the spacecraft in $\mathcal{F}_{H}$, i.e., $\psi$ is defined as an angle and not a distance. From inspection of Fig. 4 we see that $\alpha_{z}$ is solely dependent on the relative inclination, $\Delta i$, of the companion and reference orbits. The expression for $\alpha_{z}$ in terms of $\Delta_{i}$ is 


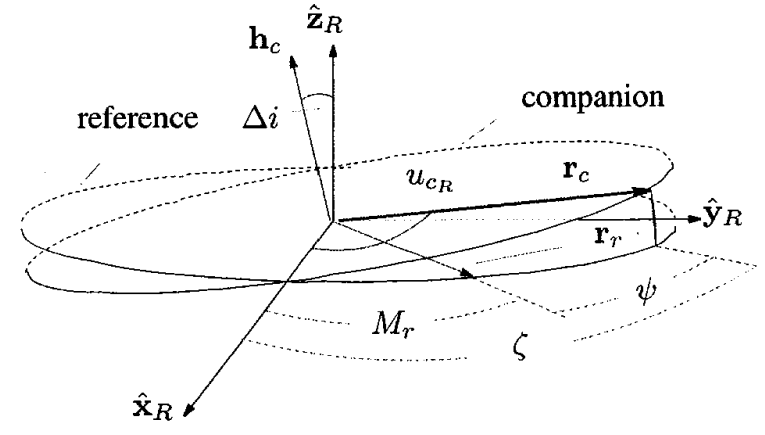

Figure 4: Geometry and Variable Definitions in $\mathcal{F}_{R}$

simply

$$
\alpha_{z}=\Delta_{i}
$$

For close formations the along-track relative motion is almost entirely dependent on the eccentricity of the companion orbit. The relative inclination of the planes of motion does have a small effect on the along-track relative motion. It was shown by Hughes and Hall [9] that to first order, the angular amplitude in the $y_{H}$-direction, $\alpha_{y}$, can be expressed in terms of the eccentricity only as

$$
\alpha_{y}=2 e_{c}
$$

Chichka [10] demonstrated independently that Eq. (12) is accurate to second order in $e$, and that the effects of $\Delta i$ on $\alpha_{y}$ are smaller than $\mathcal{O}\left(\Delta i^{2}\right)$.

Although Eqs. (11-12) determine the dimensions of the relative motion, the shape is still undefined. The turn-around conditions, seen in Eq.(10), are a convenient way to ensure that Type 1 motion is achieved. Type 2 motion warrants further study to determine if it can be of use to Leonardo. However, due to time constraints it has not yet been investigated.

The turn-around conditions impose constraints on the true anomaly, $\nu_{c}$, and the argument of periapsis, $\omega_{c}$, of the companion orbit. Before proceeding to develop the equations for $\nu_{c}$ and $\omega_{c}$, we define some variables. Refer to Fig. 4 for an illustration. The argument of latitude of the companion spacecraft in $\mathcal{F}_{R}$ is termed $u_{c_{R}}$. The argument of latitude of the reference spacecraft is denoted $M_{r}$ because the reference orbit is assumed to be circular. The along-track angular displacement of the companion with respect to the reference is denoted $\psi$ and the angle between the planes of motion is denoted $\Delta i$. From inspection of Fig. 4 we see that

$$
\psi=\zeta-M_{r}
$$

Using spherical trigonometry we can write

$$
\zeta=\tan ^{-1}\left(\cos \Delta i \tan u_{c}\right)
$$

and substituting into Eq.(13) yields

$$
\psi=\tan ^{-1}\left(\cos \Delta i \tan \left(u_{c}\right)\right)-M_{r}
$$

The turn-around conditions of Eq. (10) are expressed in terms of the spatial separations. In terms of the angular separations they are simply

$$
\dot{\psi}=0 \quad \text { when } \quad \zeta=0
$$

Taking the derivative of $\psi$ with respect to time and making use of that fact that $u_{c}=\omega_{c_{R}}+\nu_{c}$ we obtain

$$
\dot{\psi}=\frac{d \zeta}{d u_{c}}\left(\frac{d \omega_{c_{R}}}{d t}+\frac{d \nu_{c}}{d t}\right)-\frac{d M_{r}}{d t}
$$

Now, applying the turn-around conditions to ensure that the desired shape is obtained, we obtain the set of simultaneous equations

$$
\begin{aligned}
& \frac{d \zeta}{d u_{c}}\left(\frac{d \omega_{c_{R}}}{d t}+\frac{d \nu_{c}}{d t}\right)-\frac{d M_{r}}{d t}=0 \\
& \omega_{c_{R}}+\nu_{c}=k \pi \quad k=0,1,2 \ldots
\end{aligned}
$$

To ensure that the desired motion is obtained in the presence of $J_{2}$, we must obtain a periodic solution to Eqs. (18-19) while simultaneously satisfying the relation

$$
\begin{aligned}
\dot{\Omega}_{r} & =\dot{\Omega}_{c} \\
\dot{M}_{r} & =\dot{M}_{c}
\end{aligned}
$$

The reasoning for choosing these orbit constraints is explained momentarily.

To explore the solutions to these equations, first consider their functional dependence without considering the actual form. Recall that we are neglecting the periodic variations in $\omega_{c_{R}}$, $\Omega_{c}$, and $\nu_{c}$ and only considering the secular drifts to first order in $J_{2}$. The values of $\dot{\omega}_{c}, \dot{\Omega}_{c}$, and $\dot{M}_{c}$ are dependent on $a_{c}, e_{c}$, and $i_{c}$. However, from Eq. (12), to achieve the desired alongtrack dimension, the eccentricity is fixed. Therefore, only $a_{c}$, and $i_{c}$ can be varied. Furthermore, to obtain a desired across track dimension we must have a specific $\Delta i$. Fortunately, because $\Delta i=f\left(i_{r}, i_{c}, \Delta \Omega\right)$, we can vary $i_{c}$ and still maintain the required $\Delta i$ by choosing the appropriate $\Delta \Omega$. We are interested in finding $a_{c}, i_{c}, \omega_{c_{o}}$, and $\nu_{c_{o}}$ that result in a periodic solution to Eqs. (18-19) and permanently satisfy Eqs. (20-21) where the period of the solution is not necessarily equal to the Keplerian period. The variables $\omega_{c_{o}}$, and $\nu_{c_{o}}$ are the values of $\omega_{c_{R}}$, and $\nu_{c}$ at the initial epoch.

A rigorous pursuit of all the solutions is a topic of current research. However, we investigate a special case here. A interesting solution involves neglecting the secular drift in $\omega_{c_{R}}$ in the orbit design and compensating for it in the control system design. Then Eqs. (18-21) reduce to

$$
\begin{aligned}
\frac{d \zeta}{d \nu_{c}} \frac{d \nu_{c}}{d M_{c}} \frac{d M_{c}}{d t}-\frac{d M_{r}}{d t} & =0 \\
\omega_{c_{R}}+\nu_{c} & =k \pi \quad k=0,1,2 \ldots \\
\dot{\Omega}_{r} & =\dot{\Omega}_{c} \\
\dot{M}_{r} & =\dot{M}_{c}
\end{aligned}
$$


where $\omega_{c_{R}}$ is now considered constant. Equations (24-25) are uncoupled from Eqs. (22-23) and can be solved independently. See Appendix 1 for a derivation of the conditions to satisfy Eqs. (24-25). Solving for $d \zeta / d u_{c}$ from Eq. (14) we obtain

$$
\frac{d \zeta}{d u_{c}}=\frac{\cos \Delta i \sec ^{2} u_{c}}{1+\cos ^{2} \Delta i \tan ^{2} u_{c}}
$$

Evaluating this expression using Eq. (23) and substituting into Eq. (22) gives

$$
\cos \Delta i \frac{d \nu_{c}}{d M_{c}} \frac{d M_{c}}{d t}-\frac{d M_{r}}{d t}=0
$$

The mean anomaly rates are equal so we obtain

$$
\cos \Delta i \frac{d \nu_{c}}{d M_{c}}-1=0
$$

A second order expansion, found in Vallado [11], for $\nu_{t}$ in terms of $e_{c}$ and $M_{t}$ is

$$
\nu_{t}=M_{t}+2 e_{c} \sin M_{t}+\frac{5 e_{c}^{2}}{4} \sin 2 M_{t}
$$

where the subscript " $\mathrm{t}$ " is used denote the value satisfying the turn-around conditions. Substituting Eq. (29) into Eq. (28) and solving for $\cos M_{t}$ we obtain

$$
\cos M_{t}=\frac{-2 \pm \sqrt{50 e_{c}^{2}+20 / \cos \Delta i-16}}{10 e_{c}}
$$

There are two solutions for $\cos M_{t}$. For physically realistic values of $e_{c}$, we choose the positive root to ensure that the absolute value of $\cos M_{t}$ is less than one. Taking the inverse cosine will yield two solutions for $M_{t}$ as expected because there are two unique solutions to the turn-around conditions. The turn-around point is only dependent on $e_{c}$ and $\Delta i$. From inspection of Eqs. (11-12) we can write $e_{c}=\alpha_{y} / 2$ and $\Delta i=\alpha_{z}$. So, the companion spacecraft "turns around" at a specific mean anomaly and that mean anomaly is only dependent on the dimensions of the relative motion!

To satisfy the turn-around conditions, we must ensure that $\omega_{c_{R}}+$ $\nu_{t}=k \pi(\mathrm{k}=0,1, \ldots)$, in $\mathcal{F}_{R}$. From Eq. (30), we know the mean anomaly to satisfy the constraint on $\dot{\psi}$. We must ensure that the argument of periapsis, expressed in $\mathcal{F}_{R}$, satisfies the relation

$$
\omega_{c_{R}}=-\nu_{t}+k \pi \quad k=0,1,2, \ldots
$$

Only $\mathrm{n}=0,1$ provide physically unique solutions. Moreover, the $n=1$ solution is equivalent to the $n=0$ solution except that it is advanced $180^{\circ}$ in its orbit, i.e. the formation is on the other side of the Earth. The important result here is that there is only one value of $\omega_{c_{R}}$ that satisfies the turn-around conditions and provides unique formation dynamics. More importantly though, if the argument of periapsis changes from this value the shape of the relative motion will change! This will have some negative effects on the $\Delta V$ required to maintain the formation in the presence of $\mathrm{J} 2$.
Summarizing the results to this point, to obtain a formation of dimensions $\alpha_{y}$ and $\alpha_{z}$ and satisfy Eqs. (24-25) we know that

$$
\begin{aligned}
e_{c} & =\alpha_{y} / 2 \\
\Delta i & =\alpha_{z} \\
M_{t} & =\cos ^{-1}\left(\frac{-2+\sqrt{50 e_{c}^{2}+20 / \cos \Delta i-16}}{10 e_{c}}\right)(34) \\
\omega_{c_{R}} & =-\nu_{t} \quad\left(\text { in } \mathcal{F}_{R}\right)
\end{aligned}
$$

To obtain the true anomaly at turn-around, the mean anomaly from Eq. (34) can be substituted into Eq. (29)or we can solve Kepler's eq.

The remaining elements to be determined are $a_{c}, i_{c}, \Omega_{c}$ and $\omega_{c}$ in $\mathcal{F}_{I}$. Note that the $\omega_{c_{R}}$ in Eq. (35) is the argument of periapsis of the companion orbit in $\mathcal{F}_{R}$. The remaining elements can be solved using the conditions given in Appendix 1 and simple spherical geometry.

From Appendix 1 we know $\Delta a$ and $\Delta i$ to satisfy the mean anomaly and node rate constraints simultaneously.

$$
\begin{aligned}
a_{c} & =a_{r}+\Delta a \\
i_{c} & =i_{r}+\Delta i_{c}
\end{aligned}
$$

Note that $\Delta i_{c}$ is the difference in inclination between the reference and companion orbits and is not to be confused with $\Delta i$, the angle between the reference and companion orbit normal vectors.

From inspection of Fig. 5 and spherical geometry [12] we can write

$$
\Omega_{c}=\Omega_{r}+\Delta \Omega
$$

where

$$
\Delta \Omega=\cos ^{-1}\left(\frac{\cos \alpha_{z}-\cos i_{r} \cos i_{c}}{\sin i_{r} \sin i_{c}}\right)
$$

The two solutions for $\Delta \Omega$ provide two unique relative motion solutions. However, for the remainder of this work the firstquadrant solution is assumed. Although the approach is the same regardless of which solution is used, some of the following expressions are affected by the choice.

From inspection of Fig. 5 we can write

$$
\omega_{c_{I}}=\phi_{c}-\nu_{t}
$$

where $\phi_{c}$ is the arclength, in the companion plane of motion, between the Earth's equator and the line of relative nodes:

$$
\cos \frac{\phi_{c}}{2}=\sqrt{\frac{-\cos \left(\Sigma+i_{c}\right) \cos \left(\Sigma-\alpha_{z}\right)}{\sin i_{c} \sin \alpha_{z}}}
$$

where $\Sigma$ is given by

$$
\Sigma=\left(\alpha_{z}+i_{r}-i_{c}+\pi\right) / 2
$$




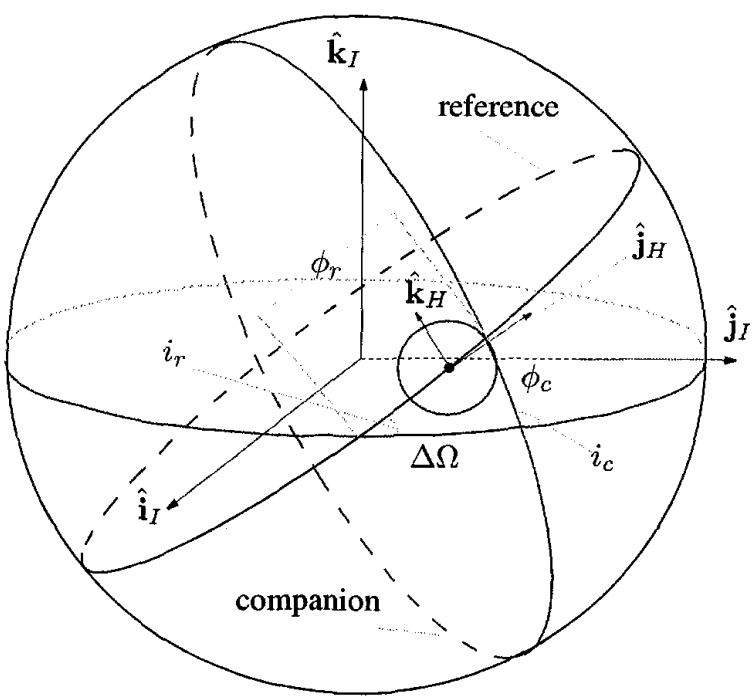

Figure 5: Geometry of the Planes of Motion

and the first or second quadrant solution for $\phi_{c}$ must be used in Eq. (40).

This completes the solution of the companion orbital elements. However, to simplify the analysis we have assumed that the companion spacecraft is at the point specified by the turnaround conditions. To complete the analysis, we must ensure that the reference spacecraft is at the appropriate point in its orbit to obtain the desired relative motion. From inspection of Fig. 5, we see this is accomplished by choosing

$$
u_{r}=\phi_{r}-\alpha_{y}=2 \cos ^{-1} \sqrt{\frac{\cos \left(\Sigma-i_{r}\right) \cos \left(\Sigma-\alpha_{z}\right)}{\sin i_{r} \sin \alpha_{z}}}-\alpha_{y}
$$

where we choose the first or second quadrant solution.

In this section we developed an algorithm to determine the state of a companion orbit, given the plane of motion of the reference circular orbit, to satisfy geometry constraints given by $\alpha_{y}$ and $\alpha_{z}$ and the relations $\dot{\Omega}_{c}=\dot{\Omega}_{r}$ and $\dot{M}_{c}=\dot{M}_{r}$. To do so we chose a point in the reference plane that greatly simplifies the algebra. The point in the reference plane will change, although only slightly, with the dimensions of the relative orbit. Therefore, if relative orbits of differing dimensions are desired, care must be taken to propagate the states to a common point in the reference orbit to obtain the correct states for the formation.

\section{Physics of the Relative Motion Solutions}

Recall that for a given plane of motion for the companion orbit, there are two solutions for the true anomaly at turn around $\nu_{t}$. Also, to satisfy the orbit constraints, for a given reference orbit, there are only two allowable planes of motion for the companion orbit. Therefore, at a given instant there are only four solutions that provide the desired relative geometry while simultaneously satisfying the mean anomaly and node rate constraints.

The four solutions are defined using Table 1. A three dimensional plot has been included of the relative motion paths of all four solutions for one orbit period (Fig. 6). The plot was created using $\alpha_{y}=1^{\circ}$ and $\alpha_{z}=1^{\circ}$. Note that there are only two distinct trajectories and two of the solutions share one trajectory while the other two share the second trajectory.

\begin{tabular}{ccc}
\multicolumn{3}{c}{ Table 1: Solution Definitions } \\
\hline \hline Solution Label & $\nu_{t}$ quadrant & $\bar{\Delta} \bar{\Omega}$ quadrant \\
\hline A & II & I \\
B & II & IV \\
C & IV & I \\
D & IV & IV \\
\hline
\end{tabular}

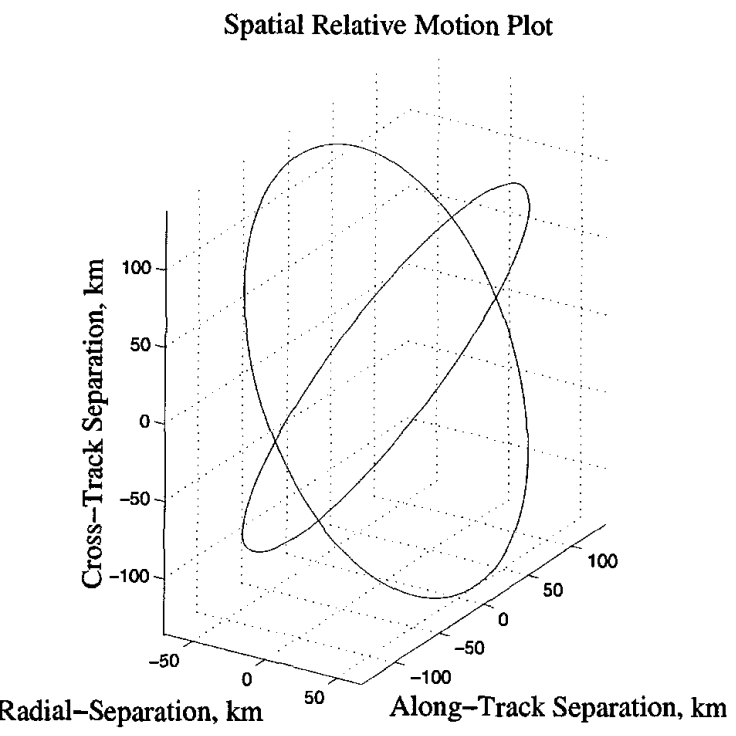

Figure 6: 3-D View of the Four Solutions

Figure 7 illustrates the projection of each type of motion in the $y_{H^{-}} z_{H}$ plane for half of an orbit. The four illustrations indicate the spacecraft positions at the four different times labeled. We see that spacecraft A and D move in the same direction on their trajectory and are about 180 degrees out of phase with each other. This is also true for B \& C. However, while A \& D move counter-clockwise, $\mathrm{B} \& \mathrm{C}$ move clockwise.

\section{Hill's Equations Approach}

To get a more intuitive understanding of the problem, we investigate another method based on Hill's equations as a first guess of the solution. We show that the results are in good agreement with the non-linear approach developed above, even 


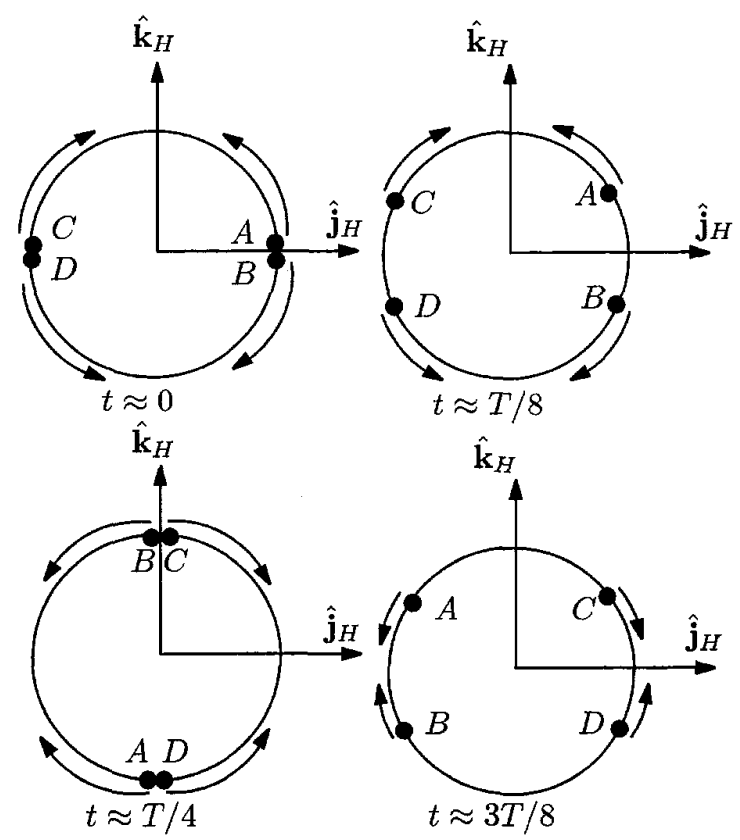

Figure 7: Physics of the Four Relative Motion Solutions

for large formations.

The Hill Clohessy Wiltshire (HCW) equations are a first order approximation of the relative motion of a spacecraft with respect to a circular reference orbit. The equations are accurate for spacecraft with separations on the order of meters to kilometers. However, large view zenith angles require large spacecraft separations (up to $3,000 \mathrm{~km}$ range). Therefore, the Hill's equations solutions must be refined using an iterative process so that the constraints are met. In their most general form, the $\mathrm{HCW}$ equations are expressed in $\mathcal{F}_{H}$ as

$$
\begin{gathered}
x(t)=A_{x y} \cos \left(n t+\phi_{x y}\right)-2 A / n \\
y(t)=-2 A_{x y} \sin \left(n t+\phi_{x y}\right)-3 A t+B \\
z(t)=A_{z} \cos \left(n t+\phi_{z}\right)
\end{gathered}
$$

where $n$ is the mean motion given by

$$
n=\sqrt{\frac{\mu}{a^{3}}}
$$

and $\mu$ is the Earth's gravitational parameter and $a$ is the semimajor axis of the reference orbit.

When specialized to the case where the pursuer spacecraft has the same orbital period as the reference, the solutions take on the form

$$
\begin{gathered}
x(t)=A_{x y} \cos \left(n t+\phi_{x y}\right) \\
y(t)=-2 A_{x y} \sin \left(n t+\phi_{x y}\right)+B \\
z(t)=A_{z} \cos \left(n t+\phi_{z}\right)
\end{gathered}
$$

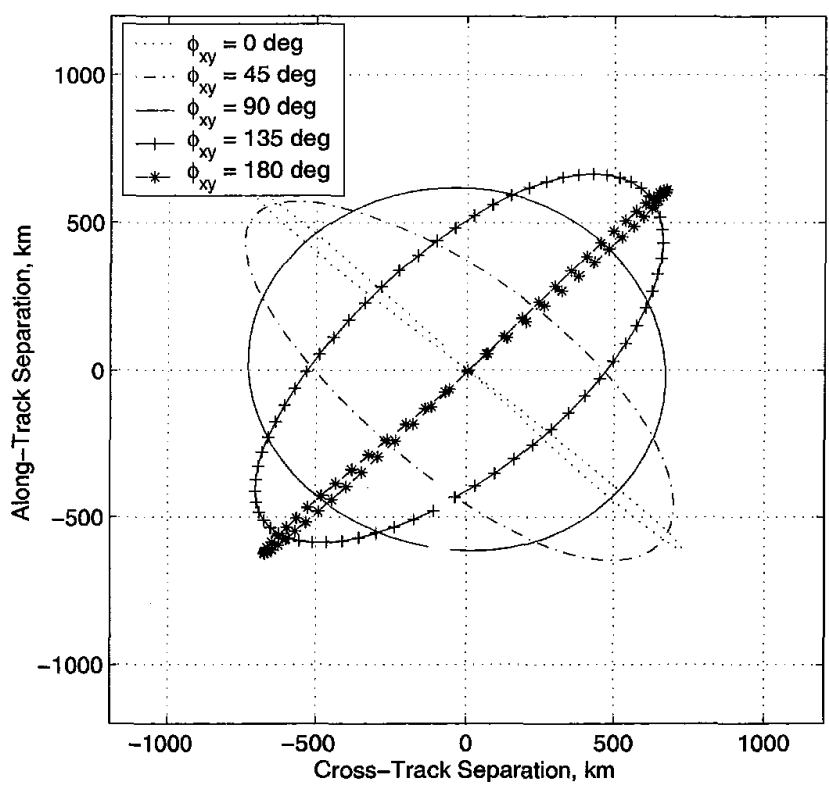

Figure 8: Relative Motion Shape vs. $\phi_{x y}$

$A_{x y}$ and $A_{z}$ characterize the amplitude or size of the relative motion. A and B define the "center" of the relative motion in $\mathcal{F}_{H}$. For this study, A and B are chosen to be zero so that the relative motion is centered on the reference.

For small amplitudes of the relative motion, the following relations between the HCW's amplitudes and the Keplerian orbital elements can be established [13]:

$$
\begin{gathered}
A_{x y}=a \cdot e_{c} \\
A_{z}=a \cdot \Delta \Omega \cdot \sin i_{r}
\end{gathered}
$$

Knowing the desired amplitudes and the reference inclination, we can determine $\Delta \Omega$ and $e_{c}$ from Eqs. (51-52). Next, we need to relate the phases $\phi_{x y}$ and $\phi_{z}$ to the node rate constraint and the turn-around conditions. The z-phase controls the initial out-of-plane distance. For nearly polar orbits, $|\Delta \Omega|$ is mainly determined by $A_{z}$. Thus, the z-phase primarily determines the sign of $\Delta \Omega$ and the inclination. For a given relative motion size, there are only four values of $\phi_{z}$ that satisfy the node rate constraint.

The xy-phase primarily determines the shape of the projection of the relative motion in the yz-plane by changing the location at which $\dot{y}(t)=0$ occurs as shown in Fig. 8. In terms of Keplerian elements, we observe that for nearly polar orbits, a change in xy-phase induces a change in argument of perigee and true anomaly. Therefore, we can infer that a change in argument of perigee will lead to a change of shape.

We wish to achieve a Type 1 relative motion described in Fig. 3. To obtain the desired relative motion geometry, we must ensure that the turn around conditions in Eq. (10) are satisfied. 
Hence, we must simultaneously satisfy the equations

$$
\begin{gathered}
\dot{y}(t)=-2 \cdot n \cdot A_{x y} \cos \left(n t_{o}+\phi_{y x}\right)=0 \\
z(t)=A_{z} \cdot \cos \left(n t_{o}+\phi_{z}\right)=0
\end{gathered}
$$

From Eqs. (53-54), the xy-phase and the z-phase can be related by

$$
\phi_{x y}=n \cdot \pi+\phi_{z} \quad n \in Z
$$

To find the proper phasing, we perform a parametric scan of the z-phase so that a Sun-synchronous node rate is achieved and use Eq. (55) as a first-guess for the xy-phase. For large amplitudes, we observe a non-centered z-motion not predicted by the HCW model. Consequently, the value of $\phi_{x y}$ obtained from Eq. 55 , is refined to obtain a relative motion as close as possible to a Type 1 solution. Table (2) presents the four solutions obtained using the HCW linear algorithm. $\phi_{z}^{*}$ and $\phi_{x}^{*}$ are the $z$-phase and $x y$-phase values found for solution $A$. The remaining solutions' phases are expressed as function of $\phi_{z}^{*}$ and $\phi_{x}^{*}$. Solutions with positive $\dot{x}_{o}$ corresponds to an argument of perigee of about $0^{\circ}$. When $\dot{x}_{o}$ is negative the argument of perigee is about $180^{\circ}$. The $\dot{z}_{0}$ velocity indicates whether $\Delta \Omega$ is positive or negative.

Table 2: HCW's Four Relative Motion Solutions

\begin{tabular}{ccccccc}
\hline \hline Solution & $\dot{z}_{o}$ & $\dot{x}_{o}$ & $\phi_{x y}$ & $\phi_{z}$ & $\Delta \Omega$ & $\omega$ \\
\hline $\mathrm{A}$ & + & + & $\phi_{x y}^{*}$ & $\phi_{z}^{*}$ & + & $\approx 0^{\circ}$ \\
$\mathrm{B}$ & - & + & $\phi_{x y}^{*}$ & $180+\phi_{z}^{*}$ & - & $\approx 0^{\circ}$ \\
$\mathrm{C}$ & + & - & $-\phi_{x y}^{*}$ & $180-\phi_{z}^{*}$ & + & $\approx 180^{\circ}$ \\
$\mathrm{D}$ & - & - & $-\phi_{x y}^{*}$ & $-\phi_{z}^{*}$ & - & $\approx 180^{\circ}$ \\
\hline \hline
\end{tabular}

Table (3) summarizes the relationship between the HCW parameters and the orbital elements for nearly polar orbits.

Table 3: HCW parameters vs. Keplerian Elements

\begin{tabular}{ccc}
\hline \hline Keplerian & HCW & Constraints \\
\hline $\mathbf{a}$ & $\mathrm{A}$ & Difference in Period \\
$\mathrm{e}$ & $A_{x y}$ & xy-dimension \\
$\mathbf{i}$ & $\phi_{z}$ & Node Rate \\
$\Omega$ & $A_{z}$ & z-dimension \\
$\omega / \nu$ & $\phi_{x y} / \mathrm{B}$ & Relative Motion Shape \\
\hline
\end{tabular}

To conclude, the HCW approach provides results in agreement with the non-linear method. The HCW equations are expressed directly in terms of the relative motion geometry. They offer an insight into the relationship between the Keplerian elements and the relative motion parameters. Although the HCW equations provide a good first-guess of the solutions, the linear approach is used here as a validation tool rather than a rigorous and reliable method. However, it works surprisingly well for large formations.

\section{Formation PERFORMANCE}

In this section we examine the performance the of the formation in light of the science requirements. The allowable formation solutions determined in section 3 are used to find specific orbits that simultaneously satisfy the science and orbit dynamics constraints. We begin by determining a reference orbit for the formation. Then, the formation geometry that best satisfies the science requirements is determined. Interestingly, if the aspect ratio of the relative motion $\alpha_{y} / \alpha_{z}$ or $A_{x y} / A_{z}$ is carefully chosen, then constant view zenith angles can be maintained over the regions of interest. Finally, we investigate the strengths and weaknesses of the formation in light of the BRDF science requirements.

\section{Orbit Design to Meet Science Requirements}

To satisfy the science requirements, the reference orbit must be Sun-synchronous with a noon equator-crossing. The orbit design approach assumes a circular reference orbit. In order to have a noon equator crossing, an epoch must be chosen. We arbitrarily choose Jan. 1, 2000 00:00:00:0000 as the reference epoch. A summary of the orbital elements for the reference are found in Table 4 . The choice of semimajor axis is complicated and discussed later. The inclination is determined from Eq. (5) with the physical constants defined by the Joint Gravity Model (JGM-2) [11]. For Leonardo, there is no scientific benefit in having a spacecraft at the reference, hence the reference spacecraft is virtual.

Viewing Angle Considerations - To effectively perform the science the formation must maintain constant view zenith angles, $\theta$, and provide sampling in view azimuth, $\phi$, to obtain an accurate estimate of the average dependence of the albedo on $\phi$.

We begin by investigating the formation's ability to provide a constant view zenith angle. For the preliminary study we choose to investigate a formation to provide zenith angles of $26^{\circ}, 37^{\circ}$, and $46^{\circ}$ degrees. Again, these angles are not consistent with any of the quadrature techniques discussed in section 2. The purpose of this preliminary investigation is to determine the feasibility of achieving constant view zenith angles using a formation of spacecraft.

Recall from Eq. (32), that the along-track dimensions of the relative motion are proportional to the eccentricity. We can write the altitude variation, $\Delta h$, of a particular orbit in formation as

$$
\Delta h=r_{\boldsymbol{a}}-r_{p}=a \alpha_{y}
$$

where $r_{a}$ and $r_{p}$ are the radii of apoapsis and periapsis respectively. From Eq. (56), we see that any formation with non-zero along-track motion will experience a variation in altitude over the course of an orbit. Moreover, the larger the relative orbit, the larger the altitude variation. 


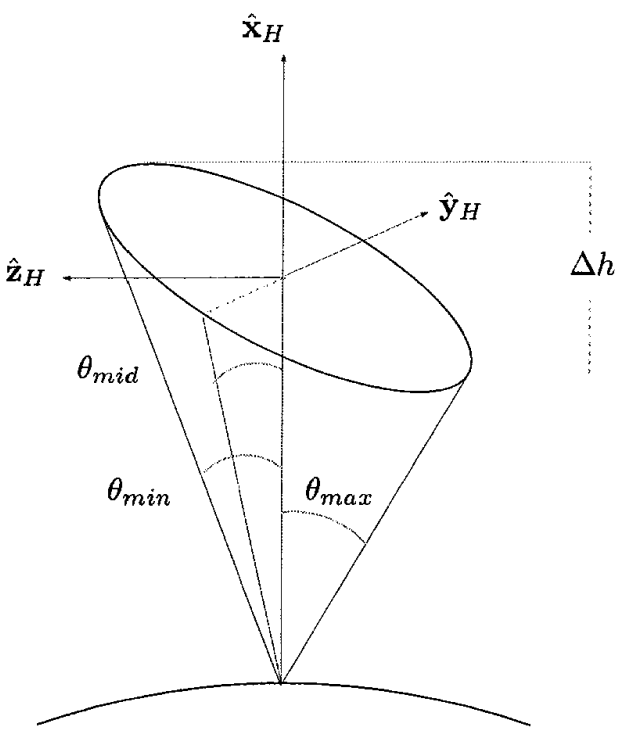

Figure 9: Altitude Variation and Viewing Geometry

Fig. 9 illustrates the altitude variation and its affect on viewing geometry. Due to the altitude variation, the view zenith angle is not constant for a circular formation defined by $\alpha_{y}=\alpha_{z}$. This is because the angular dimensions $\alpha_{y}$ and $\alpha_{z}$ are defined with the vertex at the center of the Earth. The vertex for the view zenith angle is an object at TOA. However, it is possible to adjust the aspect ratio $\alpha_{y} / \alpha_{z}$ to obtain a nearly constant $\phi$ for a portion of the orbit. The approach taken here is to design the formation so that $\phi_{\max } \approx \phi_{\text {mid }}$. This is done using a simple trial and error approach. Further study is required to determine a functional relationship between $\alpha_{y}$ and $\alpha_{z}$ to obtain the desired aspect ratio.

The altitude variation described by Eq. (56) causes some other difficulties in the orbit design. To obtain a set of view zenith angles, we must have several spacecraft in concentric relative orbits of differing dimensions. Since $\alpha_{y}$ is different for the different relative orbits, they have different altitude variations and hence experience significantly different drag forces. For small formations, the differential drag is not as significant as the differential affects of $J_{2}$. However, to achieve large view zenith angles, on the order of $50^{\circ}$, the altitude variation is on the order of $1300 \mathrm{~km}$ for a formation with $a=7600 \mathrm{~km}$ ! Hence, the reference semi-major axis must be chosen so that the formation is either entirely above the existing atmosphere or only in the outermost region. Otherwise, there will be a significant relative drift due to the differing drag forces experienced by different spacecraft. For the preliminary analysis, we require that all spacecraft remain above $500 \mathrm{~km}$. The overall design is a complicated trade-off between the desired view zenith angles, the required formation aspect ratio, and the semimajor axis. The choice of semimajor axis is driven by the largest relative orbit. To achieve a view zenith angle of $46^{\circ}$ and have a periapsis above $500 \mathrm{~km}$, the semimajor axis should be at least $7600 \mathrm{~km}$. With the reference orbit completely defined, the companion orbits can be determined to achieve the desired view zenith angles. Tables 4 and 5 summarize respectively the orbit properties for the non-linear and linear methods. The orbits are labeled "Leo1", "Leo2", and "Leo3' and are designed to achieve zenith angles of $26^{\circ}, 37^{\circ}$, and $46^{\circ}$ respectively.

Table 4: Formation Orbit Properties - Non-Linear Method

\begin{tabular}{ccccc}
\hline & Ref & Leo1 & Leo2 & Leo3 \\
\hline$\alpha_{y}$ & NA & $5.15^{\circ}$ & $8.3^{\circ}$ & $11.55^{\circ}$ \\
$\alpha_{z}$ & NA & $5.45^{\circ}$ & $8.9^{\circ}$ & $12.75^{\circ}$ \\
$a$ & $7600 \mathrm{~km}$ & $7600 \mathrm{~km}$ & $7600 \mathrm{~km}$ & $7600 \mathrm{~km}$ \\
$e$ & 0 & .045 & .072 & .101 \\
$i$ & $100.51^{\circ}$ & $100.47^{\circ}$ & $100.40^{\circ}$ & $100.29^{\circ}$ \\
$\omega$ & undef. & $180.95^{\circ}$ & $181.54^{\circ}$ & $182.141^{\circ}$ \\
$\Omega$ & $278.85^{\circ}$ & $284.39^{\circ}$ & $287.90^{\circ}$ & $291.82^{\circ}$ \\
$\nu$ & undef. & $264.90^{\circ}$ & $261.81^{\circ}$ & $258.67^{\circ}$ \\
$u$ & $90^{\circ}$ & $85.85^{\circ}$ & $83.35^{\circ}$ & $80.81^{\circ}$ \\
\hline \hline
\end{tabular}

Note: The difference in $a$ is on the order of meters and is not seen in the table.

Table 5: Formation Orbit Properties - Linear Method

\begin{tabular}{|c|c|c|c|c|}
\hline & Ref & Leol & Leo2 & Leo3 \\
\hline$\overline{A_{x y}}$ & NA & $306 \mathrm{~km}$ & $490 \mathrm{~km}$ & $665 \mathrm{~km}$ \\
\hline$A_{z}$ & NA & $702 \mathrm{~km}$ & $1180 \mathrm{~km}$ & $1700 \mathrm{~km}$ \\
\hline$\phi_{x y}$ & NA & $92.35^{\circ}$ & $95^{\circ}$ & $97.7^{\circ}$ \\
\hline$\phi_{z}$ & NA & $265.59^{\circ}$ & $263.02^{\circ}$ & $260.67^{\circ}$ \\
\hline$a$ & $7600 \mathrm{~km}$ & $7600 \mathrm{~km}$ & $7600 \mathrm{~km}$ & $7600 \mathrm{~km}$ \\
\hline$e$ & 0 & .0407 & .0667 & .0937 \\
\hline$i$ & $100.51^{\circ}$ & $100.49^{\circ}$ & $100.43^{\circ}$ & $100.34^{\circ}$ \\
\hline$\omega$ & undef. & $181.14^{\circ}$ & $180.60^{\circ}$ & $180.12^{\circ}$ \\
\hline$\Omega$ & $278.85^{\circ}$ & $285.11^{\circ}$ & $288.62^{\circ}$ & $292.27^{\circ}$ \\
\hline$\nu$ & undef. & $265.40^{\circ}$ & $263.75^{\circ}$ & $262.17^{\circ}$ \\
\hline$u$ & $90^{\circ}$ & $86.541^{\circ}$ & $84.364^{\circ}$ & $82.29^{\circ}$ \\
\hline
\end{tabular}

A plot of the relative motion is shown in Fig. 10. The solid black lines represent the actual relative orbits of the companion spacecraft. The dashed lines represent the projection of the motion into the local horizontal plane. The enormous size of the relative orbits is apparent by looking at the scale of the axes. The largest relative orbit has a diameter of over 3000 $\mathrm{km}$ !

Results- Recall that we are not concerned with the formation geometry on the dark side of the Earth. Because a Sunsynchronous orbit maintains a constant orientation with respect to the Sun, the formation can be designed to provide useful geometry only on the Sun-lit side. Furthermore, we are only interested in latitudes within $\pm 60^{\circ}$. In the following plots, the viewing angle is set to zero if the formation is outside of \pm $60^{\circ}$ latitude. The time has been normalized on the orbit period. 


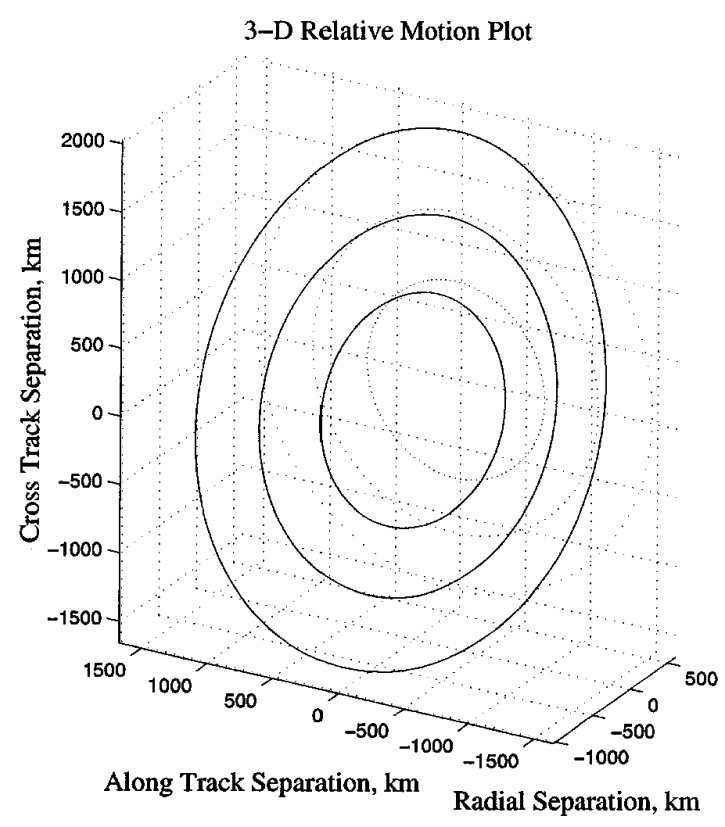

Figure 10: Relative Motion in 3-D

The view zenith angle, $\theta$, is defined as the angle between the line of sight and the local vertical of the target. Recall that according to the quadrature approach, we need to maintain specific values of the view zenith angle. Although we cannot maintain a view zenith angle over an entire orbit, it is possible to maintain the angle within the latitude band of $\pm 60^{\circ}$ as seen in Fig. 11. This is accomplished by adjusting the aspect ratio of the relative motion projection. Although there are four relative motion solutions that provide a specific value of $\theta$ and satisfy the orbit dynamics constraints, only two of the solutions can be used to obtain the desired $\theta$ on the Sun-lit portion of the Earth. The difference in the two configurations is that they are $180^{\circ}$ out of phase in the view azimuth angle. A significant problem with the formation is that initializing and maintaining high view zenith angles results in prohibitively large fuel expenditure as is demonstrated in the next section. Zenith angles above $25^{\circ}$ are probably infeasible with the $\Delta V$ allowance for Leonardo-BRDF.

The view azimuth angle, $\phi$, is defined as the angle between $y_{H}$-axis and the projection of the line of sight into the local horizontal plane. Recall that according to the quadrature approach the spacecraft must be spaced somewhat evenly in $\phi$. This is because only the average dependence of the albedo on view azimuth is used to calculate the flux integral. The azimuth angle evolution for the orbits is illustrated in Fig. 12. The azimuth angle rotates through $360^{\circ}$ over one orbit. However, as discussed earlier, for a relative orbit of given dimensions there are only four unique relative motion solutions that satisfy the geometry and node rate constraints. Because we are

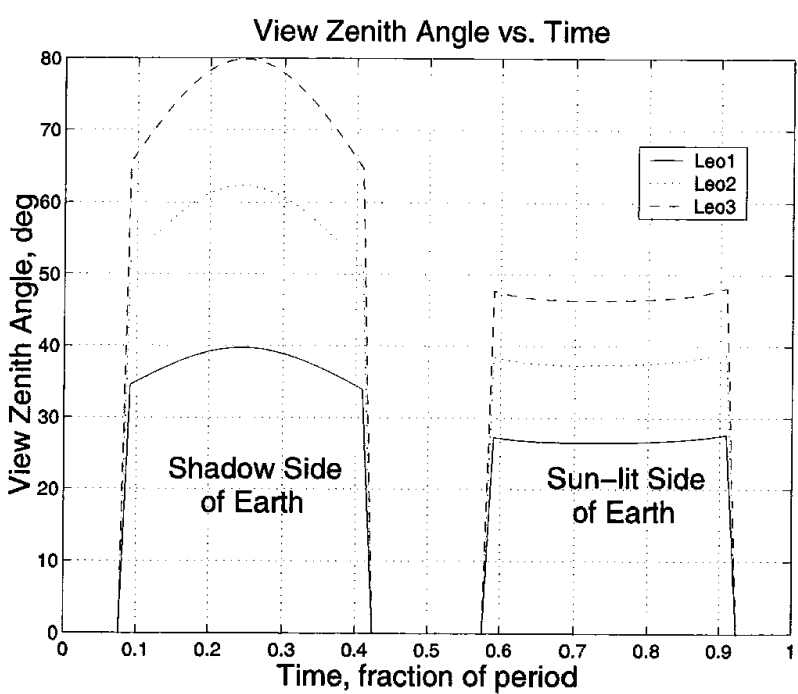

Figure 11: View Zenith Angle Evolution

only interested in the Sun-lit portion of the Earth, we can only use one pair of the solutions. The spacecraft within each pair are separated by approximately 180 degrees in $\phi$. Therefore, it is only possible to have two $\phi$ values that are equally spaced for a ring of given dimensions.

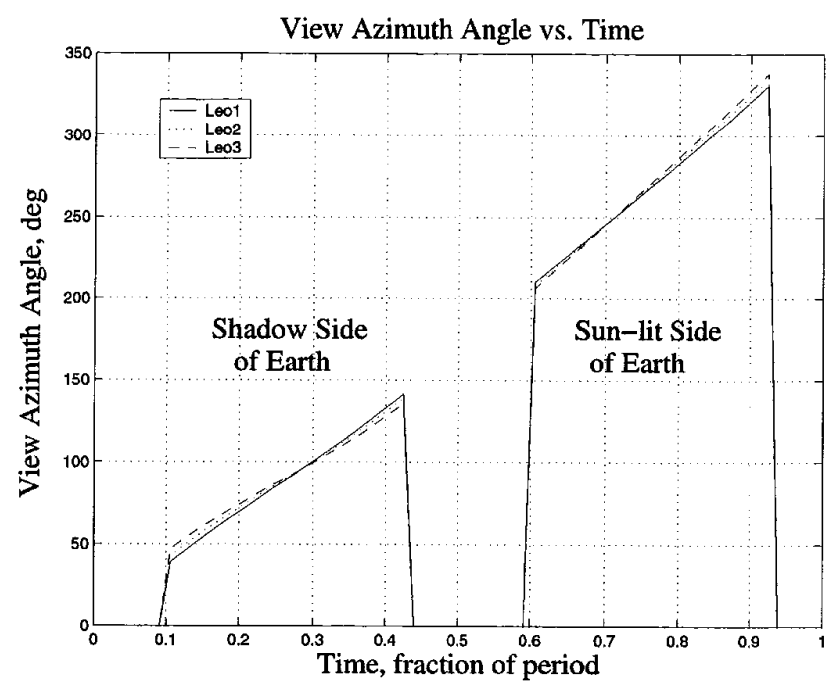

Figure 12: View azimuth Angle Evolution

In the next section we investigate strategies to initialize and maintain the formation. The approach is a simple impulsiveburn method intended to give first estimates of the $\Delta V$ 's.

\section{5. $\triangle \mathrm{V}$ BUDGET}

The total $\Delta V$ is a significant measure of the feasibility of the mission. The spacecraft are envisioned to be small, light, 
and simple. A total mission $\Delta V$ on the order of $400 \mathrm{~m} / \mathrm{s}$ per spacecraft is expected. We assume all spacecraft will be launched on the same vehicle into a common orbit. First, a common launch orbit is chosen so that the initialization maneuvers are distributed among the spacecraft within the array. Then, we develop an optimal Lambert scheme to find the minimum two-impulsive-burn initialization $\Delta V$. Once the array is initialized, frequent, small maneuvers are necessary to maintain the desired formation geometry. Perturbations such as the Earth's oblateness (mainly J2) and atmospheric drag induce secular drifts between the spacecraft which, if not corrected, will cause formation separation. Consequently, we develop maintenance strategies based on work by Chao [4].

\section{Formation Initialization}

In this section we investigate a general two-impulse orbit initialization maneuver strategy based on Lambert's problem. For single-satellite missions, the main concern is to achieve the final desired orbit. For multiple-satellite missions the spacecraft must reach the desired final position at the correct time so that the desired array configuration is achieved. In general, a simple satellite rendezvous sequence depends on three parameters: the initial orbit state at the first maneuver, the time of flight, and the target state. For a formation of spacecraft, the final state is completely determined by the initial orbit state and the time of flight. Hence, we lose a degree of freedom and can expect to have larger $\Delta V$ 's for initializing formation flying missions. To minimize the fuel consumption, we consider the orbit initialization sequence of each spacecraft independently. Consequently, the transfer from the parking orbit to the final orbit will occur at different times for different spacecraft. The formation is not considered "initialized" until the last spacecraft joins the array.

An illustration of the orbit geometry of the initial and final states is shown in Fig. 13. In general, the orbits are not coplanar but they have been drawn as coplanar orbits for simplicity. All spacecraft are assumed to be launched into the Leo 2 orbit. This allows a sharing of the $\Delta V$ between the spacecraft Leo1 and Leo3. Leo2 will require no maneuver to achieve the desired orbit. The position $\mathbf{r}_{c_{o}}$ is the initial position of the companion spacecraft in the parking orbit at the initial epoch. The position $\mathbf{r}_{c_{f}}$ is the desired position of the companion spacecraft after a time of flight $t_{f}$. The initial position of the companion spacecraft, had it been in the appropriate orbit at the initial epoch, is denoted $\mathbf{r}_{t_{o}}$. It is important to realize that $\mathbf{r}_{t_{o}}$ is completely determined by $\mathbf{r}_{c_{o}}$ and the relative motion solutions of Section 3. For a given initial epoch we know both vectors $\mathbf{r}_{c_{o}}$ and $\mathbf{r}_{t_{o}}$. For a given time of flight we can propagate $\mathbf{r}_{t_{o}}$ to determine the value of $\mathbf{r}_{c_{f}}$. Then the $\Delta V$ can be found by solving Lambert's problem. By varying the initial epoch and the time of flight we can search for the optimal twoimpulsive-burn initialization maneuver sequence. The results are seen in Table 6.

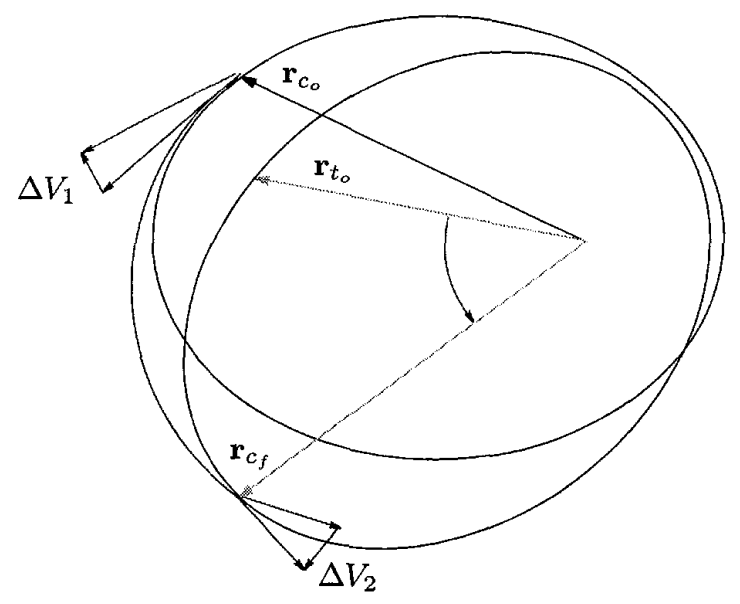

Figure 13: Initialization Maneuver Approach

The maneuvers to initialize the formation are prohibitively high for the scenarios chosen. The estimates may be lowered by investigating alternative parking orbits and using more than two impulses. However, it is probable that rotating formations providing view zenith angles of more than $25^{\circ}$ are infeasible within the constraints of Leonardo.

Table 6: Formation Initialization $\Delta V$

\begin{tabular}{cc}
\hline \hline Orbit & $\Delta V m / s$ \\
\hline Leo1 & 475.32 \\
Leo2 & 0 \\
Leo3 & 536.67 \\
\hline \hline
\end{tabular}

\section{Formation Maintenance}

In general, perturbing forces from atmospheric drag, non-spherical affects, solar radiation pressure and third body effects will cause dramatic changes in the formation geometry over short time scales. Control strategies to maintain the formation in the presence of these perturbations can take on many forms. In this work we investigate a simple impulsive burn strategy to obtain some preliminary estimates. More sophisticated state feedback techniques and primer vector theory are topics of future research and are expected to reduce the $\Delta V$ required to maintain the formation.

In section 3, we investigate a formation design approach that suggests if the periapsis of the companion orbit drifts from its initial value, the shape of the relative orbit will change. The affect of the mean anomaly drift of the companion orbit is not as problematic because the reference mean anomaly drifts as well. We are only concerned with the relative mean anomaly drift. Therefore, the maneuver strategy focuses on maintaining the argument of periapsis. Chao et al. [4] developed a method to find the optimal locations of two symmetrical tangential burns to change the argument of periapsis without changing 
the other orbital elements. The true anomaly to provide an optimal maneuver depends on the desired change in argument of periapsis, and the eccentricity:

$$
\nu_{o p t}= \pm\left(\cos ^{-1}(-e) \pm e+\frac{\Delta \omega}{2}\right)
$$

Maintenance maneuvers are performed daily to correct for the argument of perigee, semi-major axis and eccentricity drift. These corrections are applied at the initial mean anomaly and the reference is set back to its initial state. This technique only provides a rough estimation of the necessary maintenance $\Delta V$.

Table 7: Rotating Formation Maintenance $\Delta V$

\begin{tabular}{cc}
\hline \hline Orbit & $\Delta V m / s / d a y$ \\
\hline Leo1 & 5.8 \\
Leo2 & 9.7 \\
Leo3 & 13.8 \\
\hline \hline
\end{tabular}

Table 7 shows that the maintenance $\Delta V$ budget for the formation is highly expensive. Again, the technique used is not optimal and other techniques, such as Primer Vector Theory and Linear Quadratic Regulator, are yet to be investigated, but are expected to improve the maintenance budget.

\section{Alternative Formation}

The science requirements for Leonardo favor a Sun-synchronous formation. However, due to the large fuel expense required to initialize and maintain the Sun-synchronous formation we investigate an alternative formation. Specifically, the dynamics of the problem presented in a previous section suggest that considerable fuel savings may be afforded by using orbits at the critical inclination. For critically inclined orbits the secular argument of periapsis drift is zero. The impact of the new formation geometry on science has not been investigated. The dynamics and control of a critically inclined formation is presented in an attempt to explore more realistic possibilities for formations on the scale of Leonardo. The states for the critical formation are presented in Table 8 . The values for $\alpha_{y}$ and $\alpha_{z}$ to achieve the desired viewing angles were determined by trial and error.

Table 8: Formation Orbit Properties: Non-Linear Method

\begin{tabular}{ccccc}
\hline & Ref & Leo1 & Leo2 & Leo3 \\
\hline$\alpha_{y}$ & NA & $4.95^{\circ}$ & $7.5^{\circ}$ & $10.1^{\circ}$ \\
$\alpha_{z}$ & NA & $5.45^{\circ}$ & $9.05^{\circ}$ & $12.9^{\circ}$ \\
$a$ & $7600 \mathrm{~km}$ & $7600 \mathrm{~km}$ & $7600 \mathrm{~km}$ & $7600 \mathrm{~km}$ \\
$e$ & 0 & .0432 & .0654 & .0881 \\
$i$ & $63.435^{\circ}$ & $63.542^{\circ}$ & $63.680^{\circ}$ & $63.879^{\circ}$ \\
$\omega$ & undef. & $177.52^{\circ}$ & $176.20^{\circ}$ & $174.83^{\circ}$ \\
$\Omega$ & $278.85^{\circ}$ & $284.94^{\circ}$ & $288.96^{\circ}$ & $293.24^{\circ}$ \\
$\nu$ & undef. & $264.81^{\circ}$ & $261.79^{\circ}$ & $258.63^{\circ}$ \\
$u$ & $90^{\circ}$ & $82.32^{\circ}$ & $77.98^{\circ}$ & $73.46^{\circ}$ \\
\hline
\end{tabular}

As expected the $\Delta V$ to initialize the critically inclined formation are still considerably high. The values are shown in Table 9. The same initialization strategy used for the Sunsynchronous formation is used here. The spacecraft are assumed to be launched into the Leo 2 orbit from a common launch vehicle. Leo1 and Leo3 are maneuvered into their appropriate orbits from the Leo 2 orbit. For this approach no fuel expense is required to initialize Leo2.

Table 9: Formation Initialization $\Delta V$

\begin{tabular}{cc}
\hline Orbit & $\Delta V \mathrm{~m} / \mathrm{s}$ \\
\hline Leo1 & 489.46 \\
Leo2 & 0 \\
Leo3 & 521.47 \\
\hline
\end{tabular}

As explained earlier, the argument of perigee drift is the main driver of the maintenance $\Delta V$ cost. Using a critically inclined reference orbit improves the maintenance $\Delta V$ considerably. Table 10 presents the maintenance $\Delta V$ values for the formation. The improvement is on the order of 70 times less fuel, compared to the sun-synchronous formation, to maintain the Leo1 orbit and 30 times less fuel to maintain the Leo3 orbit.

Table 10: Rotating Formation Maintenance $\Delta V$

\begin{tabular}{cc}
\hline \hline Orbit & $\Delta V m / s / d a y$ \\
\hline Leo1 & 0.085 \\
Leo2 & 0.204 \\
Leo3 & 0.483 \\
\hline
\end{tabular}

Critically inclined formations suffer from one difficulty that is avoided by using Sun-synchronous formations. A Sun-synchronous orbit, by definition, rotates at the same rate as the Earth rotates about the Sun. Hence a Sun-synchronous formation will maintain a constant geometry with respect to the Sun However, a critically inclined formation will change its orientation with respect to the Sun. For Leonardo we wish to make measurements of the reflected Sunlight and these measurements can only be made on the side of the orbit that can provide the appropriate geometry. Therefore two spacecraft will be needed to ensure a specific viewing geometry is maintained over the life of the mission. However, there are some scientific advantages in the critically inclined formation. The Sun position will change with respect to the reference plane and a much more general understanding of the effect of illumination geometry can be obtained.

\section{CONCLUSIONS}

A preliminary flight dynamics analysis was presented for Leonardo-BRDF. The orbit dynamics constraints, driven by the science requirements, are extremely difficult to achieve in a fuel efficient manner. To accurately estimate the radiative forcing using a quadrature approach, the formation must provide 
specific, constant view zenith angles and a nearly equal separation in view azimuth. To achieve these requirements we developed a design approach using orbital elements that simultaneously satisfies geometry and orbital constraints. We showed that it is possible to maintain specific view zenith angles for at least half of an orbit. However, there are only two solutions among four possible solutions that provide the desired view zenith angles on the Sun-lit portion of the Earth. The azimuth angle constraint is not satisfied. Indeed, the azimuth angle history is fully determined by the view zenith angle constraints and the reference orbit. We foresee that a combination of the rotating formation and alternative formation geometries could provide better radiative forcing approximations. The $\Delta V$ 's necessary to establish and maintain the formation were estimated using some preliminary impulsive maneuver strategies. The fuel expenditure, especially for maintenance, was found to be prohibitively high for a Sun-synchronous formation. Consequently, we investigated a formation designed using a critically-inclined reference orbit. The new maintenance $\Delta V$ 's were on the order of 70 times lower for certain orbits in the formation. Future designs will focus on lowering both the initialization and maintenance $\Delta V$ s by carefully choosing the launch orbit and reference orbit. In addition to improvements in the orbit design, the maneuver strategies could be improved by using optimal control approaches.

\section{REFERENCES}

[1] John Houghton et.al., "The Science of Climate Change," Second Assessment Report of the Intergovernmental Panel on Climate Change (IPCC), Cambridge Univ. Press, 1996.

[2] Hanspeter Schaub and Kyle T. Alfriend, " $J_{2}$ Invariant Relative Orbits for Spacecraft Formation Flying," Proceedings of the Flight Mechanics Symposium, NASA/GSFC, May, 1999.

[3] C.C.Chao and J.E. Pollard and S.W.Janson, "Dyanmics and Control of Cluster Orbits for Distributed Space Missions," AIAA Space Flight Mechanics Meeting, Breckneridge, Colorado, Feb., 1999.

[4] C.C. Chao and G.E. Peterson and M.D. Menn, "Formationkeeping Strategies for GEO Halo Collocation," AIAA/AAS Astrodynamics Specialist Conference and Exhibit, Denver, Colorado, August, 1999.

[5] B. Wielicki and R. Cess and M. King and D. Randall and E. Harrison, "1995: Mission to Planet Earth: role of clouds and radiation in climate," Bull. Amer. Meteor. Soc. $76,1995$.

[6] Milton Abramowitz and Irene Stegun, "Handbook of mathematical functions with formulas, graphs, and mathematical tables ," New York: Dover Publications, 1955.
[7] George Hill, "Researches in the Lunar Theory,"American Journal of Mathematics 1, 5-26, 1878.

[8] W. H. Clohessy and R.S. Wiltshire, "Terminal Guidance System for Satellite Rendezvous," Journal of the Aerospace Sciences 27(9), 653-658, 1960.

[9] Steven P. Hughes and Christopher D. Hall, "Optimal Configurations of Rotating Spacecraft Formations," Proceedings of the Richard H. Battin Astrodynamics Symposium, College Station, Texas, Mar., 2000.

[10] David F. Chichka, "Dynamics of Clustered Satellites via Orbital Elements," AIAA/AAS Astrodynamics Specialist Conference and Exhibit, Denver, Colorado, August, 1999.

[11] David A. Vallado, "Fundamentals of Astrodynamics and Applications,” New York: McGraw Hill, 1997.

[12] James R. Wertz, "Spacecraft Attitude Determination and Control," Dordrecht: Kluwer Academic Publishers, 1978.

[13] James L. Garrison, Thomas G. Gardner and Penina Axelrad, " Relative Motion in Highly Elliptical Orbits," AAS/AIAA Spaceflight Mechanics Meeting, Albuquerque, New Mexico, February, 1995.

Steven Hughes is an Aerospace Engineer at Goddard Space Flight Center where he has worked in the Guidance, Navigation, and Control Center since January 2000. His current research is in formation flying dynamics and control. Before GSFC, Mr. Hughes was at Virginia Tech where he received his B.S. and M.S. in Aerospace Engineering in 1997 and 1999 respectively.

Laurie Mailhe is an Mission Analyst at a.i. solutions, Inc. where she has worked on trajectory and mission design since January 2000. Her current research is in formation flying $d y$ namics and trajectory optimization. She received her aerospace engineer diploma from EPF, Paris in 1998 and her M.S. in Aerospace and Aeronautics from Purdue university in 1999. 


\section{Appendix 1}

Here we present a second order solution in $\Delta a$ and $\Delta i_{c}$ to ensure that the mean anomaly rates and node rates respectively, are equal for the reference and companion orbits in the presence of first order $J_{2}$ effects. Note that $\Delta i_{c}$ is the differnce in inclination of the reference and companion orbits and not to be confused with $\Delta i$, the angle between the orbit normal vectors of the reference and companion planes of motion. In general $\dot{\Omega}=f(a, e, i)$. Recall that we assume a circular reference orbit. Also, for a given formation dimension the eccentricity of the companion orbit is defined, to second order, by equation Eq. (12). So the following is a system of two second order equations in two unknowns. The exact expressions for $\dot{M}$ and $\dot{\Omega}$, to first order in $J_{2}$, are given by Eqs. (5) and (7). In general to second order we have

$$
\begin{aligned}
\dot{\Omega}_{c} & \approx \dot{\Omega}_{r e f}+\left.\frac{\partial \dot{\Omega}}{\partial a}\right|_{r e f} \Delta a+\left.\frac{\partial \dot{\Omega}}{\partial i}\right|_{r e f} \Delta i_{c}+\left.\frac{\partial \dot{\Omega}}{\partial e}\right|_{r e f} \Delta e \\
& +\left.\frac{1}{2} \frac{\partial^{2} \dot{\Omega}}{\partial a^{2}}\right|_{r e f} \Delta a^{2}+\left.\left.\frac{1}{2} \frac{\partial^{2} \dot{\Omega}}{\partial \dot{i}^{2}}\right|_{r e f} \Delta i_{c}^{2} \frac{1}{2} \frac{\partial^{2} \dot{\Omega}}{\partial e^{2}}\right|_{r e f} \Delta e^{2} \\
& +\left.\frac{\partial^{2} \dot{\Omega}}{\partial a \partial i}\right|_{r e f} \Delta a \Delta i_{c}+\left.\frac{\partial^{2} \dot{\Omega}}{\partial a \partial e}\right|_{r e f} \Delta a \Delta e+\left.\frac{\partial^{2} \dot{\Omega}}{\partial e \partial i}\right|_{r e f} \Delta e \Delta i_{c}
\end{aligned}
$$

$$
\begin{aligned}
\dot{M}_{c} & \approx \dot{M}_{r e f}+\left.\frac{\partial \dot{M}}{\partial a}\right|_{r e f} \Delta a+\left.\frac{\partial \dot{M}}{\partial i}\right|_{r e f} \Delta i_{c} \\
& +\left.\frac{\partial \dot{M}}{\partial e}\right|_{r e f} \Delta e+\left.\frac{1}{2} \frac{\partial^{2} \dot{M}}{\partial a^{2}}\right|_{r e f} \Delta a^{2}+\left.\frac{1}{2} \frac{\partial^{2} \dot{M}}{\partial i^{2}}\right|_{r e f} \Delta i_{c}^{2} \\
& +\left.\frac{1}{2} \frac{\partial^{2} \dot{M}}{\partial e^{2}}\right|_{r e f} \Delta e^{2}+\left.\frac{\partial^{2} \dot{M}}{\partial a \partial i}\right|_{r e f} \Delta a \Delta i_{c}+\left.\frac{\partial^{2} \dot{M}}{\partial a \partial e}\right|_{r e f} \Delta a \Delta e \\
& +\left.\frac{\partial^{2} \dot{M}}{\partial e \partial i}\right|_{r e f} \Delta e \Delta i_{c}
\end{aligned}
$$

where the reference conditions are defined by the reference orbit properties

$$
a_{r e f}=a_{r} \quad e_{r e f}=0 \quad i_{r e f}=i_{r}
$$

Before presenting the solutions for $\Delta a$ and $\Delta i_{c}$, we examine the relative magnitudes of some of the terms in the expansion for $\dot{\Omega}_{c}$. Specifically, the derivative of $\dot{\Omega}_{c}$ with respect to $a$ can be written

$$
\frac{\partial \dot{\Omega}_{c}}{d a}=\frac{21}{4} \frac{n R_{e}^{2} J_{2}}{a p^{2}} \cos i=-\frac{7}{2 a} \dot{\Omega}_{c}
$$

The coefficient, $-7 / 2 a$ is of interest here. Working in SI units we know $a>6500 \mathrm{~km}$ for practical orbits and $J_{2} \approx .001082$, a dimensionless quantity. Therefore, $|-7 / 2 a|$ is smaller than $\mathcal{O}\left(J_{2}\right)$. Because $J_{2}$ also appears in the $\dot{\Omega}$ equation we see that

$$
\frac{\partial \dot{\Omega}_{c}}{d a} \approx \mathcal{O}\left(J_{2}^{2}\right)
$$

Therefore in Eq. (58) we neglect the terms involving derivatives with respect to $a$ because they are higher order terms.
The resulting expression is

$$
\begin{aligned}
& \dot{\Omega}_{c} \approx \dot{\Omega}_{r e f}+\left.\frac{\partial \dot{\Omega}}{\partial i}\right|_{r e f} \Delta i_{c}+\left.\frac{1}{2} \frac{\partial^{2} \dot{\Omega}}{\partial i^{2}}\right|_{r e f} \Delta i_{c}^{2}+ \\
& \left.\frac{\partial \dot{\Omega}}{\partial e}\right|_{r e f} \Delta e++\left.\frac{1}{2} \frac{\partial^{2} \dot{\Omega}}{\partial e^{2}}\right|_{r e f} \Delta e^{2}+\left.\frac{\partial^{2} \dot{\Omega}}{\partial e \partial \dot{i}}\right|_{r e f} \Delta e \Delta i_{c}
\end{aligned}
$$

There are two distinct advantages to this simplification. First the algebraic form of the solutions will likely be much simpler. Secondly, we have decoupled the node rate equation from the mean anomaly rate equation and the system of second order equations can now be solved in sequence rather than simultaneously. Because we have assumed a circular reference and $e_{c}$ is given by Eq. (12), we can write $\Delta e=e_{c}$. Solving Eq. (63) for $\Delta i_{c}$, when $\dot{\Omega}_{c}-\dot{\Omega}_{r e f}=0$, and choosing the physically meaningful of the two solutions we obtain

$$
\Delta i_{c}=\sec i\left(-\sin i+\sqrt{4 e_{c}^{2} \cos ^{2} i+\sin ^{2} i}\right)
$$

Now we can solve Eq. (59), when $\dot{M}_{c}-\dot{M}_{\text {ref }}=0$, for $\Delta a$ where $\Delta i_{c}$ and $\Delta e$ are known. The solution is

$$
\Delta a=\frac{-B-\sqrt{B^{2}-4 A C}}{2 A}
$$

where

$$
\begin{aligned}
A= & 40 a^{2}+63 c_{e}(1+3 \cos 2 i) \\
B= & -4 a\left(8 a^{2}+7 c_{e}+21 c_{e}\left(\cos 2 i-2 \Delta i_{c} \sin 2 i\right)(67)\right. \\
C= & 12 a^{2} c_{e}\left(e_{c}^{2}+\left(3 e_{c}^{2}-4 \Delta i_{c}^{2}\right) \cos 2 i\right. \\
& \left.-4 \Delta i_{c} \sin 2 i\right) \\
c_{e}= & J_{2} R_{e}^{2}
\end{aligned}
$$

Again the physically meaningful solution of the quadratic equation is used.

Determining the range of validity of these approximate solutions is non-trivial and will depend on the size of the relative orbit and properties of the reference orbit. However, the approximations are excellent for the formations investigated here which are on the order of $3000 \mathrm{~km}$ in diameter.

\section{Appendix 2}

Appendix 2 presents a summary of the four relative motion solutions equations discussed in section 3 . The solutions are generated for a given reference plane of motion and the desired angular dimensions, $\alpha_{y}$ and $\alpha_{z}$, of the relative orbit. The quantities, $a_{r}, i_{r}$, and $\Omega_{r}$ of the circular reference orbit are assumed known. However, the position of the reference spacecraft in the reference orbit is chosen to simplify the algebra. Therefore $u_{r}$, the argument of latitude of the reference orbit, is determined below. Care must be taken to propagate the the states of all spacecraft in formation to a common point in the reference orbit. 


$$
\begin{aligned}
& \text { Table 11: Solution A \& C } \\
& \text { Solution Quadrant } \\
& \text { A } \quad M_{c} \text { in II } \\
& \text { C } \quad M_{c} \text { in III } \\
& \text { A \& } \mathrm{C} \quad \Delta \Omega \text { in I } \\
& a_{c}=a_{r}+\Delta a \quad \text { (See App. 1) } \\
& e_{c}=\alpha_{y} / 2 \\
& i_{c}=i_{r}+\Delta i_{c} \quad \text { (See App. 1) } \\
& \Delta \Omega=\cos ^{-1}\left(\frac{\cos \alpha_{z}-\cos i_{r} \cos i_{c}}{\sin i_{r} \sin i_{c}}\right) \\
& \Omega_{c}=\Omega_{r}+\Delta \Omega \\
& M_{c}=\cos ^{-1} \frac{-2+\sqrt{50 e_{c}^{2}+20 / \cos \alpha_{z}-16}}{10 e_{c}} \\
& \Sigma=\frac{\left(\alpha_{z}+i_{r}-i_{c}+\pi\right)}{2} \\
& \phi_{r}=2 \cos ^{-1} \sqrt{\frac{\cos \left(\Sigma-i_{r}\right) \cos \left(\Sigma-\alpha_{z}\right)}{\sin i_{r} \sin \alpha_{z}}} \\
& \phi_{c}=2 \cos ^{-1} \sqrt{\frac{-\cos \left(\Sigma+i_{c}\right) \cos \left(\Sigma-\alpha_{z}\right)}{\sin i_{c} \sin \alpha_{z}}} \\
& \omega_{c}=\phi_{c}-\nu_{c} \\
& u_{r}=\phi_{r}-\alpha_{y} \quad \text { For Solution A } \\
& u_{r}=\phi_{r}+\alpha_{y} \quad \text { For Solution C } \\
& a_{c}=a_{r}+\Delta a \quad \text { (See App. 1) } \\
& e_{c}=\alpha_{y} / 2 \\
& i_{c}=i_{r}+\Delta i_{c} \quad \text { (See App. 1) } \\
& \Delta \Omega=\cos ^{-1}\left(\frac{\cos \alpha_{z}-\cos i_{r} \cos i_{c}}{\sin i_{r} \sin i_{c}}\right) \\
& \Omega_{c}=\Omega_{r}+\Delta \Omega \\
& M_{c}=\cos ^{-1} \frac{-2+\sqrt{50 e_{c}^{2}+20 / \cos \alpha_{z}-16}}{10 e_{c}} \\
& \Sigma=\frac{\left(\alpha_{z}+i_{c}-i_{r}+\pi\right)}{2} \\
& \phi_{r}=2 \cos ^{-1} \sqrt{\frac{-\cos \left(\Sigma+i_{r}\right) \cos \left(\Sigma-\alpha_{z}\right)}{\sin i_{r} \sin \alpha_{z}}} \\
& \phi_{c}=2 \cos ^{-1} \sqrt{\frac{\cos \left(\Sigma-i_{c}\right) \cos \left(\Sigma-\alpha_{z}\right)}{\sin i_{c} \sin \alpha_{z}}} \\
& \omega_{c}=\phi_{c}-\nu_{c} \\
& u_{r}=\phi_{r}-\alpha_{y} \quad \text { For Solution B } \\
& u_{r}=\phi_{r}+\alpha_{y} \quad \text { For Solution D }
\end{aligned}
$$

\begin{tabular}{cc}
\hline \hline Solution & Quadrant \\
\hline $\mathrm{B}$ & $M_{c}$ in II \\
$\mathrm{D}$ & $M_{c}$ in III \\
$\mathrm{B} \& \mathrm{D}$ & $\Delta \Omega$ in IV \\
\hline \hline
\end{tabular}

